\title{
m-normal theories
}

\author{
by \\ Ludomir Newelski (Wrocław)
}

\begin{abstract}
Originally, m-independence, $\mathcal{M}$-rank, m-stability and m-normality were defined only for small stable theories. Here we extend the definitions to an arbitrary small countable complete theory. Then we investigate these notions in the new, broader context. As a consequence we show that any superstable theory with $<2^{\aleph_{0}}$ countable models is m-normal. In particular, any $*$-algebraic group interpretable in such a theory is abelian-by-finite.
\end{abstract}

0. Introduction. Throughout, $T=T^{\mathrm{eq}}$ is a complete countable firstorder theory and we work within a monster model $\mathfrak{C}$ of $T$. Also we assume that $T$ is small, that is, $S_{n}(\emptyset)$ is countable for every $n$.

In a series of papers [Ne1-Ne4, Ne6] I investigated the notion of multiplicity of a type in a superstable theory with $<2^{\aleph_{0}}$ (that is, few) countable models. This led me to introduce several new notions, like m-independence, $\mathcal{M}$-rank, m-stability and so on. I believe that these notions, apart from their relevance to Vaught's conjecture, may be useful in model theory in general.

In this paper the definitions of these notions are extended to the case of an arbitrary complete countable small theory. In Section 1 we exhibit basic properties of these notions. In the next sections we investigate m-normal theories and $*$-algebraic groups interpretable in such theories. Still the most important case for us remains the superstable theories with few countable models. As a consequence of our results on arbitrary m-normal theories we show that any superstable theory with few countable models is m-normal. In particular, any $*$-algebraic group interpretable in such a theory is abelianby-finite. The most natural example of a $*$-algebraic group is the group $G / G^{0}$ for a given (standard) group $G$ interpretable in $T$.

1. Preliminaries. While originally $\mathcal{M}$-rank and related notions were defined in a small stable theory for types and finite tuples of standard ele-

2000 Mathematics Subject Classification: Primary 03C45.

This paper was written during my visit to MSRI, Berkeley, in the Spring of 1998.

Research supported by KBN grant 2 P03A 00609. 
ments of $\mathfrak{C}$, in $[\mathrm{Ne} 4]$ I noticed that the same definitions work for a broader class of objects, called $*$-finite tuples. Then it turned out that a special kind of $*$-finite tuples, called $*$-algebraic tuples, are central for the theory of multiplicities. For instance, to compute the $\mathcal{M}$-rank of any type it is enough to compute the $\mathcal{M}$-rank of a corresponding $*$-algebraic type.

It turns out that if we restrict our attention just to *-algebraic tuples, then all the definitions work in an arbitrary countable small theory. In this section we recall the notions of $*$-finite and $*$-algebraic tuple and introduce the definitions of m-independence and related notions for such tuples within an arbitrary theory $T=T^{\mathrm{eq}}$.

Definition 1.1. A $*$-finite tuple is a tuple $a_{I}=\left\langle a_{i}, i \in I\right\rangle$ of elements of $\mathfrak{C}$, with the index set $I$ countable, such that $a_{I}$ is contained in the definable closure $\operatorname{dcl}\left(a^{\prime}\right)$ for some $a^{\prime} \in \mathfrak{C}$.

Notice that if $T$ is small and $a_{I}$ is $*$-finite, then $S_{n}\left(a_{I}\right)$ is countable. In this paper $A, B, C, \ldots$ will denote finite sets of $*$-finite tuples of elements of $\mathfrak{C}$ and $a, b, c \ldots$ will denote $*$-finite tuples. To distinguish between $*$-finite tuples and elements of $\mathfrak{C}$, we call the elements of $\mathfrak{C}$ standard.

For any $*$-finite tuple $a_{I}$ and any set $A$ we define the type $\operatorname{tp}\left(a_{I} / A\right)$ (in variables $\left.x_{I}=\left\langle x_{i}, i \in I\right\rangle\right)$ in a natural way. $\mathrm{A} *$-finite type is a type whose realizations are $*$-finite. $S_{I}(A)$ denotes the set of complete types over $A$ in variables $x_{I}$. If $p\left(x_{I}\right)$ is a type over $A$, then $S_{I}(A) \cap[p]$ denotes the set of complete types in $S_{I}(A)$ containing $p$. For $p \in S_{I}(A), p(\mathfrak{C})$ denotes the set of realizations of $p$. Also, in this paper $a \equiv b(A)$ means that $\operatorname{tp}(a / A)=$ $\operatorname{tp}(b / A)$.

Remark 1.2. Assume $T$ is small, $A \subseteq B$ are finite and $p\left(x_{I}\right) \in S_{I}(A)$ is $*$-finite. Then $S_{I}(B) \cap[p]$ is countable, hence the isolated points are dense in $S_{I}(B) \cap[p]$.

Proof. Easy.

Definition 1.3. (1) We say that a $*$-finite tuple $a_{I}$ is $*$-algebraic over $A$ if $a_{I} \subseteq \operatorname{acl}(A)$.

(2) A type in $S_{I}(A)$ is *-algebraic if each of its realizations is $*$-algebraic over $A$.

Sometimes we omit $A$ in the phrase "*-algebraic over $A$ " if $A=\emptyset$ or the identity of $A$ is clear from the context. Notice that if $p \in S_{I}(A)$ is *-algebraic, then $p(\mathfrak{C})$ has cardinality $\leq 2^{\aleph_{0}}$. We say that $p \in S_{I}(A)$ is algebraic if $p(\mathfrak{C})$ is finite. Clearly in this case $p$ is $*$-finite and $*$-algebraic. We say that $a_{I}$ is algebraic over $A$ if $\operatorname{tp}\left(a_{I} / A\right)$ is algebraic. Also now $\operatorname{acl}(A)$ is the set of all $*$-finite tuples algebraic over $A$. This might raise the question about the cardinality of $\operatorname{acl}(A)$. Notice that any element of a tuple in $\operatorname{acl}(A)$ 
is contained in the definable closure of $\operatorname{acl}(A)$ in the old sense [Ne4]. For an infinite set of parameters $D$ we define $\operatorname{acl}(D)$ as $\bigcup\left\{\operatorname{acl}\left(D^{\prime}\right): D^{\prime} \subset D\right.$ finite $\}$.

Assume $p \in S_{I}(A)$ is $*$-algebraic. There is a natural topology on $p(\mathfrak{C})$ generated by the base consisting of sets of the form $\left[b_{J}\right]=\left\{a_{I} \in p(\mathfrak{C})\right.$ : $\left.a_{J}=b_{J}\right\}$, where $b_{J} \subseteq \mathfrak{C}$ and $J \subseteq I$ is finite. Here $a_{J}=\left\langle a_{i}, i \in J\right\rangle$, $b_{J}=\left\langle b_{i}, i \in J\right\rangle \cdot p(\mathfrak{C})$ with this topology is homeomorphic to a closed homogeneous subset of the Cantor set.

Definition 1.4. Given any type $q\left(x_{I}\right)$ and any set $A$ of parameters, we define the trace of $q$ over $A$ as the set $\operatorname{Tr}_{A}(q)=\left\{r \in S_{I}(\operatorname{acl}(A)): r \cup q\right.$ is consistent\}.

We omit $A$ in $\operatorname{Tr}_{A}(q)$ if $A=\emptyset$. In the case of a stable theory $T$, for $p \in S_{I}(A)$, elements of $\operatorname{Tr}_{A}(p)$ are called stationarizations of $p$ over $A$ (this is because they are stationary types with respect to forking independence in stable theories). Notice that if $p \in S_{I}(A)$ is $*$-algebraic, then any $r \in \operatorname{Tr}_{A}(p)$ is realized by exactly one $I$-tuple of elements of $\mathfrak{C}$. So in this case there is a natural correspondence between $\operatorname{Tr}_{A}(p)$ and $p(\mathfrak{C})$, which is a homeomorphism. So for notational simplicity, for $a_{I} *$-algebraic over $A$ and for $B$ containing $A$, we often identify $\operatorname{Tr}_{A}\left(a_{I} / B\right)$ with the set of realizations of $\operatorname{tp}\left(a_{I} / B\right)$.

If $q \in S_{I}(B)$ is an extension of a *-algebraic type $p \in S_{I}(A)(A \subseteq B)$, then clearly either $q(\mathfrak{C})$ is nowhere dense in $p(\mathfrak{C})$ or $q(\mathfrak{C})$ is clopen in $p(\mathfrak{C})$. In the latter case we say that $q$ is an $m$-free extension of $p$. This distinction leads to the definition of the $\operatorname{rank} \mathcal{M}$ and m-independence.

Definition 1.5. (1) $\mathcal{M}$ is the minimal function defined on complete *-algebraic types, with values in Ord $\cup\{\infty\}$, such that for every complete *-algebraic $p$ and every ordinal $\alpha$ we have

$(\mathcal{M}) \quad \mathcal{M}(p) \geq \alpha+1 \Leftrightarrow$ for some $*$-algebraic $q$ extending $p, q(\mathfrak{C})$ is nowhere dense in $p(\mathfrak{C})$ and $\mathcal{M}(q) \geq \alpha$.

(2) For $a_{I}$ *-algebraic over $A$ and any finite $B$ we say that $a_{I}$ is $m$ independent of $B$ over $A\left(a_{I}{ }^{\mathrm{m}} \mathfrak{L} B(A)\right)$ if $\operatorname{tp}\left(a_{I} / A B\right)$ is an m-free extension of $\operatorname{tp}\left(a_{I} / A\right)$.

So $\mathcal{M}$-rank on $*$-algebraic types is the foundation rank for m-independence (in the m-stable case, where m-independence is well-founded).

In particular for a $*$-algebraic $p, \mathcal{M}(p)=0$ iff $p$ is algebraic, and $\mathcal{M}(p)=$ 1 iff $p(\mathfrak{C})$ is infinite, but for any complete extension $q$ of $p$, either $q$ is algebraic or $q(\mathfrak{C})$ is open in $p(\mathfrak{C})$. We say that $T$ is $m$-stable if the $\mathcal{M}$-rank of every $*$-algebraic type is $<\infty$.

REMARK 1.6. Assume $p \in S_{I}(A)$ is *-algebraic and $q \in S_{I}(B)$ extends $p$. Then $q(\mathfrak{C})$ is open in $p(\mathfrak{C})$ iff $q$ is isolated in $S_{I}(B) \cap[p]$. 
Proof. $\Rightarrow$ Assume $q(\mathfrak{C})$ is open in $p(\mathfrak{C})$. Then $q(\mathfrak{C})$ is also closed in $p(\mathfrak{C})$ and for some finite $J \subseteq I$ and finitely many tuples $a_{I}^{0}, \ldots, a_{I}^{n-1} \in$ $p(\mathfrak{C}), q(\mathfrak{C})=\bigcup_{i<n}\left[a_{J}^{i}\right]$.

Let $\varphi\left(x_{I}\right)=\bigvee_{i<n} x_{J}=a_{J}^{i}$. We see that $\varphi(\mathfrak{C}) \cap p(\mathfrak{C})=q(\mathfrak{C})$, hence $\varphi(\mathfrak{C})$ is $B$-invariant and equivalent to a formula $\varphi^{\prime}$ over $B$. Clearly, $\varphi^{\prime}$ isolates $q$ in $S_{I}(B) \cap[p]$.

$\Leftarrow$ Assume $\varphi\left(x_{I}\right)$ isolates $q$ in $S_{I}(B) \cap[p]$. Choose a finite set $J \subseteq I$ such that $\varphi=\varphi\left(x_{J}\right)$ depends on $x_{J}$ only (i.e. the $x_{i}, i \notin J$, are dummy variables in $\left.\varphi\left(x_{I}\right)\right)$. Since $p$ is $*$-algebraic, the set $\left\{a_{J}: \models \varphi\left(a_{J}\right)\right.$ and $a_{J} \subseteq a_{I}$ for some $\left.a_{I}=p\right\}$ is finite, say it consists of $a_{J}^{0}, \ldots, a_{J}^{n-1}$. Clearly $q(\mathfrak{C})=\bigcup_{i<n}\left[a_{J}^{i}\right]$, so $q(\mathfrak{C})$ is open in $p(\mathfrak{C})$.

FACT 1.7. Assume $p \subseteq q$ are $*$-algebraic.

(1) If $q(\mathfrak{C})$ is open in $p(\mathfrak{C})$, then $\mathcal{M}(q)=\mathcal{M}(p)$.

(2) If $\mathcal{M}(q)<\infty$ and $q(\mathfrak{C})$ is nowhere dense in $p(\mathfrak{C})$, then $\mathcal{M}(q)<\mathcal{M}(p)$.

Proof. This is an easy induction.

Corollary 1.8. If $T$ is small and $p \in S_{I}(A)$ is $*$-algebraic, then $p$ has an $m$-free extension in $S_{I}(B)$ for any finite $B$ containing $A$.

Proof. This follows from Remarks 1.2 and 1.6.

Assume $a_{I}$ is a $*$-finite tuple and $A \subseteq B$ are finite. The topology on $\operatorname{Tr}_{A}\left(a_{I} / A\right)$ is induced by $A$-definable finite equivalence relations. Let $\mathrm{FE}_{I}(A)$ denote the set of such equivalence relations. If $E\left(x_{J}, x_{J}^{\prime}\right) \in \mathrm{FE}_{I}(A)$ (for some finite $J \subseteq I$ ), then let $a_{E}$ be the (standard) name of the set of $E$ classes meeting $\operatorname{Tr}_{A}\left(a_{I} / B\right)$ (which is a closed subset of $\operatorname{Tr}_{A}\left(a_{I} / A\right)$ ). Clearly, the tuple $a^{+}$consisting of all such $a_{E}$ 's is *-algebraic over $A$. We call it the name of $\operatorname{Tr}_{A}\left(a_{I} / B\right)$. Similarly, the tuple $a^{*}$ consisting of elements $a_{J} / E\left(x_{J}, x_{J}^{\prime}\right), E \in \mathrm{FE}_{I}(A)$, is *-algebraic over $A$. We call it the name of $\operatorname{stp}\left(a_{I} / A\right)$.

Assume $Z \subset X \times Y, a \in X$ and $b \in Y$. The set $Z_{a}=\{y \in Y:(a, y) \in Z\}$ is called the vertical section of $Z$ at $a$, while $Z^{b}=\{x \in X:(x, b) \in Z\}$ is the horizontal section of $Z$ at $b$.

$\mathrm{m}$-independence has the following properties.

Lemma 1.9. Assume $A, B, C$ are finite sets of $*$-finite tuples and $a, b$ are $*$-algebraic over $A$.

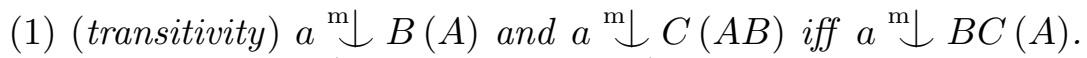

(2) (symmetry) $a \mathrm{~m}^{\mathrm{m}} b(A)$ implies $b \stackrel{\mathrm{m}}{\perp} a(A)$.

(3) If $B \in \operatorname{acl}(A)$ then $a \stackrel{\mathrm{m}}{\perp} B(A)$.

(4) If $B^{*}$ is the $*$-algebraic name of $\operatorname{stp}(B / A)$, then a ${ }^{\mathrm{m}} \downarrow B\left(A B^{*}\right)$. In particular, $a \stackrel{\mathrm{m} \downarrow}{\perp} B(A)$ iff $a{ }^{\mathrm{m}} \downarrow B^{*}(A)$ and $\mathcal{M}(a / A B)=\mathcal{M}\left(a / A B^{*}\right)$. 
(5) If $a^{+}$is the name of $\operatorname{Tr}_{A}(a / A B)$ then $a \stackrel{\mathrm{m}}{\downarrow} B\left(A a^{+}\right)$and $\mathcal{M}(a / A B)$ $=\mathcal{M}\left(a / A a^{+}\right)$.

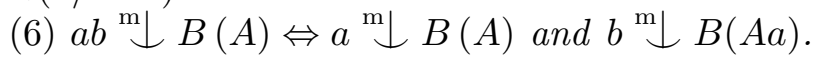

Proof. (1) is obvious. (2) Let $p=\operatorname{tp}(a / A), q=\operatorname{tp}(b / A), s=\operatorname{tp}(a b / A)$, $p_{b}=\operatorname{tp}(a / A b)$ and $q_{a}=\operatorname{tp}(b / A a)$. Then $s(\mathfrak{C})$ is a closed topological subspace of $p(\mathfrak{C}) \times q(\mathfrak{C}), p_{b}(\mathfrak{C})$ is the horizontal section of $s(\mathfrak{C})$ at $b$ and $q_{a}(\mathfrak{C})$ is the vertical section of $s(\mathfrak{C})$ at $a$. Now the following conditions are equivalent.

(a) $a \stackrel{\mathrm{m}}{\perp} b(A)$.

(b) $p_{b}(\mathfrak{C})$ is open in $p(\mathfrak{C})$.

(c) $s(\mathfrak{C})$ is open in $p(\mathfrak{C}) \times q(\mathfrak{C})$.

(d) $q_{a}(\mathfrak{C})$ is open in $q(\mathfrak{C})$.

(e) $b \stackrel{\mathrm{m}}{\perp} a(A)$.

(a) $\Leftrightarrow(\mathrm{b})$ and $(\mathrm{d}) \Leftrightarrow(\mathrm{e})$ follow from definition. (b) $\Leftrightarrow(\mathrm{c})$ and $(\mathrm{c}) \Leftrightarrow(\mathrm{d})$ follow from the fact that all the horizontal [respectively, vertical] sections of $s(\mathfrak{C})$ are conjugate and from the Kuratowski-Ulam category theorem [Ox].

(3) follows from (2) and the fact that $\operatorname{Tr}_{A}(B / A)$ is finite for $B \in \operatorname{acl}(A)$.

(4) By the choice of $B^{*}$ we have $\operatorname{tp}\left(B / A B^{*}\right) \vdash \operatorname{tp}(B / \operatorname{acl}(A))$, hence also $\operatorname{tp}\left(B / A B^{*}\right) \vdash \operatorname{tp}\left(B / A B^{*} a\right)$. By symmetry of isolation, $\operatorname{tp}\left(a / A B^{*}\right) \vdash$ $\operatorname{tp}\left(a / A B^{*} B\right)$, hence $\operatorname{Tr}_{A B^{*}}\left(a / A B^{*}\right)=\operatorname{Tr}_{A B^{*}}\left(a / A B^{*} B\right)$ and the first clause follows. Then the second clause follows from (1)-(3) and the fact that $B^{*} \in$ $\operatorname{dcl}(A B)$.

(5), (6) are left as an exercise.

(4) and (5) of Lemma 1.9 explain why $*$-algebraic tuples are central to describing $\mathrm{m}$-independence in the case of a small stable theory (in that case m-independence is defined for arbitrary $*$-finite tuples, but it is still determined by its restriction to $*$-algebraic tuples).

For $a *$-algebraic over $A$ we write $\mathcal{M}(a / A)$ for $\mathcal{M}(\operatorname{tp}(a / A))$. $\mathcal{M}$-rank on *-algebraic types satisfies Lascar-style inequalities.

FACT 1.10. Assume $a, b$ are $*$-algebraic over $A$. Then

$$
\mathcal{M}(a / A b)+\mathcal{M}(b / A) \leq \mathcal{M}(a b / A) \leq \mathcal{M}(a / A b) \oplus \mathcal{M}(b / A) .
$$

Proof. This is an easy application of the properties of m-independence from Corollary 1.8 and Lemma 1.9. The proof is similar to the case of the Lascar inequalities for $U$-rank.

2. $\mathbf{m}$-normal theories. There are some similarities between the theory of forking and the theory of m-independence. From the geometric point of view forking is most regular in a 1-based (that is, weakly normal) stable theory. In the case of m-independence, the corresponding notion is that of an m-normal theory. In this paper we will study m-normal theories. We will prove that any superstable theory with few countable models is m-normal. 
Definition 2.1. We say that $T$ is $m$-normal if for every finite $A$ and $a, b *$-algebraic over $A$, there is a $c \in \operatorname{acl}_{A}(a) \cap \operatorname{acl}_{A}(b)$ with $a \stackrel{\mathrm{m}}{\downarrow} b(A c)$.

This definition differs from the definition of m-normal theory given in [Ne4], but [Ne4, Theorem 1.10] shows that for stable $T$ both definitions are equivalent. We will generalize [Ne4, Theorem 1.10] to arbitrary small theories below.

Assume $A \subseteq B$ are finite. We say that $\operatorname{Tr}_{A}(a / B)$ is locally [almost] $A a$-invariant if for some $E \in \mathrm{FE}(A)$ the set $[E(x, a)] \cap \operatorname{Tr}_{A}(a / B)$ is fixed by $\operatorname{Aut}(\mathfrak{C} / A a)$ [respectively: there are finitely many $A a$-conjugates of this set].

We shall use the following lemma from [Ne4, Remark 0.2].

Lemma 2.2. Assume $a$ is *-algebraic over $A$ and $A \subseteq B$. Then $\operatorname{Tr}_{A}(a / B)$ is locally almost Aa-invariant iff for some $e \in \operatorname{acl}(A), \operatorname{Tr}_{A}(a / B e)$ is Aae-invariant.

The next theorem is proved in [Ne4] under the additional assumption that $T$ is stable. In the proof there we use non-forking extensions of types, which may not exist in an arbitrary small theory. So now we have to replace carefully non-forking extensions by m-free extensions of some $*$-algebraic types.

TheOREM 2.3. Assume $T$ is small. Then the following conditions are equivalent.

(1) $T$ is m-normal.

(2) For every finite $A \subseteq B$ and every $a$ *algebraic over $A, \operatorname{Tr}_{A}(a / B)$ is locally almost Aa-invariant.

(3) For every finite $A \subseteq B$ and every *-finite $a, \operatorname{Tr}_{A}(a / B)$ is locally almost Aa-invariant.

(4) For every finite $A \subseteq B \subseteq \mathfrak{C}_{=}\left(\mathfrak{C}_{=}\right.$is the "standard" sort of $\left.\mathfrak{C}^{\mathrm{eq}}\right)$ and every finite tuple a of elements of $\mathfrak{C}_{=}, \operatorname{Tr}_{A}(a / B)$ is locally almost $A$ invariant.

Proof. $(1) \Rightarrow(2)$. Let $a^{+}$be the name of $\operatorname{Tr}_{A}(a / B)$. So $a^{+}$is $*$-algebraic over $A$ and $\operatorname{Tr}_{A}(a / B)=\operatorname{Tr}_{A}\left(a / A a^{+}\right)$. By (1) choose $c \in \operatorname{acl}_{A}(a) \cap \operatorname{acl}_{A}\left(a^{+}\right)$ with $a \stackrel{\mathrm{m}}{\perp} a^{+}(A c)$. Now $\operatorname{Tr}_{A}\left(a / A a^{+} c\right)$ is clopen both in $\operatorname{Tr}_{A}(a / A c)$ and in $\operatorname{Tr}_{A}\left(a / A a^{+}\right)$. This implies that $\operatorname{Tr}_{A}\left(a / A a^{+}\right)$is locally almost $A c$-invariant, hence also locally almost $A a$-invariant.

$(2) \Rightarrow(1)$. Let $a, b$ be $*$-algebraic over $A$. Choose an $E \in \mathrm{FE}(A)$ such that the set $X=\left[E\left(x_{I}, a\right)\right] \cap \operatorname{Tr}_{A}(a / A b)$ is almost $A a$-invariant. Let $a^{+}$be the name of $X$. We see that $a^{+} \in \operatorname{acl}_{A}(a) \cap \operatorname{acl}_{A}(b)$ and $a \stackrel{\mathrm{m}}{\downarrow} b\left(A a^{+}\right)$.

$(2) \Rightarrow(3)$ and $(3) \Rightarrow(4)$ are clear.

$(4) \Rightarrow(2)$. Assume that $A \subseteq B$ are finite and $a$ is $*$-algebraic over $A$. Without loss of generality (as in the proof of $(1) \Rightarrow(2)$ ) $B$ is *-algebraic over $A$. We need to show that $\operatorname{Tr}_{A}(a / B)$ is locally almost $A a$-invariant. 
First choose finite $A^{0} \subseteq B^{0} \subseteq \mathfrak{C}_{=}$so that $A \subseteq \operatorname{dcl}\left(A^{0}\right), B \subseteq \operatorname{dcl}\left(B^{0}\right), a B \sqrt{\mathrm{m}}$ $A_{0}(A)$ and $a \stackrel{\mathrm{m} \downarrow}{ } B^{0}\left(A^{0} B\right)$. Then $a^{\mathrm{m} \downarrow} A^{0}(A)$ and $a \stackrel{\mathrm{m} \downarrow}{ } B^{0}(B)$, hence $\operatorname{Tr}_{A}\left(a / A^{0}\right)$ is clopen in $\operatorname{Tr}_{A}(a / A)$ and $\operatorname{Tr}_{A}\left(a / B^{0}\right)$ is clopen in $\operatorname{Tr}_{A}(a / B)$ and homeomorphic to $\operatorname{Tr}_{A^{0}}\left(a / B^{0}\right)$. It is enough to show that

$$
\operatorname{Tr}_{A^{0}}\left(a / B^{0}\right) \text { is locally almost } A^{0} a \text {-invariant. }
$$

Indeed, suppoose we have proved that. Since $a$ is *-algebraic over $A$, the topology on $\operatorname{Tr}_{A^{0}}\left(a / A^{0}\right)$ is induced by $\mathrm{FE}(A)$, so for some $E \in \mathrm{FE}(A)$, the set $X=[E(x, a)] \cap \operatorname{Tr}_{A^{0}}\left(a / B^{0}\right)$ is almost $A^{0} a$-invariant. Let $c$ be the name of $X$ (as a closed subset of $\operatorname{Tr}_{A}(a / A)$ ). So $c$ is $*$-algebraic over $A$ and $c \in \operatorname{acl}\left(A^{0} a\right)$. Also, since $X$ is clopen in $\operatorname{Tr}_{A}(a / B)$ we see that $c \in \operatorname{acl}(B)$. Now $B \stackrel{\mathrm{m}}{\downarrow} A^{0}(A a)$ hence $c \in \operatorname{acl}_{A a}(B) \cap \operatorname{acl}_{A a}\left(A^{0}\right)$ gives $c \in \operatorname{acl}(A a)$, i.e. $X$ is almost $A a$-invariant.

To prove (a) we may assume that $A=A^{0}$ and $B=B^{0}$, that is, that the original sets $A \subseteq B$ are subsets of $\mathfrak{C}_{=}$. Now let $b$ be a tuple of elements of $\mathfrak{C}_{=}$with $a \subseteq \operatorname{dcl}(b)$. Let $b^{*}$ be the name of $\operatorname{stp}(b / A)$, so $b^{*}$ is $*$-algebraic over $A$. By Lemma 1.9(4), $a \stackrel{\mathrm{m}}{\downarrow} b\left(A b^{*}\right)$, so $\mathcal{M}\left(a / A b^{*}\right)=0$ and $a \in \operatorname{acl}_{A}\left(b^{*}\right)$. In fact, one can prove that $a \in \operatorname{dcl}_{A}\left(b^{*}\right)$. We can choose $b$ so that $b^{*} \mathfrak{m} B(A a)$. Also, $\operatorname{Tr}_{A}(b / B)=\operatorname{Tr}_{A}\left(b^{*} / B\right)$.

By (4), $\operatorname{Tr}_{A}(b / B)$ is locally almost $A b$-invariant. Hence for some $E^{\prime} \in$ $\mathrm{FE}(A)$, the set $Y=\left[E^{\prime}\left(x, b^{*}\right)\right] \cap \operatorname{Tr}_{A}\left(b^{*} / B\right)$ is almost $A b$-invariant. Let $c^{\prime}$ be the name of $Y$. So $c^{\prime} \in \operatorname{acl}_{A}(b)$. Also, $c \in \operatorname{acl}_{A}\left(b^{*}\right)$. Then $b^{*} \mathfrak{m} B(A a)$ implies that $c^{\prime} \in \operatorname{acl}_{A}(a)$.

Let $f: \operatorname{Tr}_{A}\left(b^{*} / A\right) \rightarrow \operatorname{Tr}_{A}(a / A)$ be the definable function witnessing $a \in \operatorname{dcl}_{A}\left(b^{*}\right)$. So $f$ is an open continuous surjection and $\operatorname{Tr}_{A}(a / B)=$ $f\left[\operatorname{Tr}_{A}\left(b^{*} / B\right)\right]$. We see that $f[Y]$ is a clopen neighbourhood of $a$ in $\operatorname{Tr}_{A}(a / B)$, which is almost $A a$-invariant $\left(\operatorname{as} c^{\prime} \in \operatorname{acl}_{A}(a)\right)$. So $\operatorname{Tr}_{A}(a / B)$ is locally almost $A a$-invariant.

Condition (4) in Theorem 2.3 (or rather a condition intermediate between (3) and (4)) was given as the definition of m-normal theories in [Ne4]. We see that that definition agrees with the new one. Moreover, the current definition is easier to work with in particular applications. On the other hand, condition (4) in Theorem 2.3 seems easier to verify for a concrete theory $T$, so it may serve as a tool for proving that such a theory is m-normal. (4) is parallel to the condition " $\mathrm{Cb}(a / A) \subseteq \operatorname{acl}(a)$ " defining 1-based theories.

The following theorem has the same proof as [Ne4, Theorem 1.4], with standard types replaced by $*$-algebraic types.

THEOREM 2.4. In a small m-normal theory the $\mathcal{M}$-rank of any $*$-algebraic type is either finite or $\infty$.

COROLlary 2.5. In a small m-stable m-normal theory the $\mathcal{M}$-rank of any *-algebraic type is finite. 
I do not know of any small theory which is not m-normal. In [Ne3, Ne4] I formulated the $\mathcal{M}$-gap conjecture, saying that in a small stable theory there is no type $p$ with $\omega \leq \mathcal{M}(p)<\infty$. In order to refute this conjecture one would have to find a theory which is not m-normal.

In [Bu1] Buechler characterized 1-based theories among superstable theories of finite rank as those theories in which every $U$-rank 1 type is locally modular. We are now heading towards a similar characterization of m-normal theories of finite $\mathcal{M}$-rank (that is, m-normal m-stable theories). Such a characterization will be given in the next section. We will need the following lemma.

Lemma 2.6. Assume $T$ is $m$-stable, $A \subseteq B$ are finite and $a$ is $*$-algebraic over $A$. Then $\operatorname{Tr}_{A}(a / B)$ is invariant over $A$ and some finitely many realizations of $\operatorname{tp}(a / B)$.

Proof. Let $b$ be the name of $\operatorname{Tr}_{A}(a / B)$. Then $b$ is $*$-algebraic over $A$. We prove the lemma by induction on $\mathcal{M}(b / A)$.

CASE 1. If $\mathcal{M}(b / A)=0$, then $b \in \operatorname{acl}(A)$. Let $b=b_{0}, \ldots, b_{k}$ be all the $A$-conjugates of $b$ and let $X_{0}=\operatorname{Tr}_{A}(a / B), X_{1}, \ldots, X_{k}$ be the closed subsets of $\operatorname{Tr}_{A}(a / A)$ named by them. Choose $a_{i} \in X_{0} \triangle X_{i}$ for every $i=1, \ldots, k$. Clearly $X_{0}$ is invariant over $A a_{1} \ldots a_{k}$.

Case 2. Now suppose $\mathcal{M}(b / A)=\alpha>0$. It follows that $\operatorname{Tr}_{A}(a / B)$ is nowhere dense in $\operatorname{Tr}_{A}(a / A)$, hence $a{ }^{\mathrm{m}} \not{L} b(A)$. In particular, $\mathcal{M}(b / A a)<\alpha$. Choose $a^{\prime} \equiv a(A b)$ with $a^{\prime}{ }^{\mathrm{m}} a(A b)$. Let $A^{\prime}=A a$ and $b^{\prime}$ be the name of $\operatorname{Tr}_{A^{\prime}}\left(a^{\prime} / A^{\prime} b\right)=\operatorname{Tr}_{A}\left(a^{\prime} / A^{\prime} b\right)$.

It follows that $\left.\operatorname{Tr}_{A}\left(a^{\prime} / A^{\prime} b\right)\right)$ is clopen in $\operatorname{Tr}_{A}\left(a^{\prime} / A b\right)=\operatorname{Tr}_{A}(a / A b)$, hence $b^{\prime} \in \operatorname{acl}_{A}(b)$ and $\mathcal{M}\left(b^{\prime} / A^{\prime}\right) \leq \mathcal{M}\left(b / A^{\prime}\right)<\alpha$. By the induction hypothesis (applied to $a^{\prime}, A^{\prime}$ and $B^{\prime}=A^{\prime} b^{\prime}$ ), $b^{\prime}$ is definable over $A a$ and finitely many realizations of $\operatorname{tp}\left(a^{\prime} / A^{\prime} b^{\prime}\right)$.

But $\operatorname{Tr}_{A}(a / B)$ is a union of finitely many $A b$-conjugates of $\operatorname{Tr}_{A}\left(a^{\prime} / A^{\prime} b\right)$. Each of these conjugates is invariant over $A$ and some finitely many realizations of $\operatorname{tp}(a / B)$. So $\operatorname{Tr}_{A}(a / B)$ also has this property.

Lemma 2.6 corresponds to the fact that for every stationary type $p \in S(A)$ in a stable theory, every element of $\mathrm{Cb}(p)$ is contained in the definable closure of any infinite Morley sequence in $p$.

Even though there are strong analogies between the theory of forking and the theory of multiplicities, which suggest the way to characterize mnormality by geometric means, this task is not straightforward due to some differences between the two subjects. The main difference is the absence of "stationary" types in the case of m-independence on $*$-algebraic tuples. That is, if $a$ is $*$-algebraic over $A$, then $\operatorname{stp}(a / A)$ is realized just by $a$ alone, so there is no point in speaking of Morley sequences in it. Instead we use 
flat Morley sequences, introduced in [Ne6]. This notion makes use of an additional tool, which we are given in the case of m-independence: the set of realizations of a $*$-algebraic type carries a natural topology.

As in the theory of forking, here we have the notion of m-orthogonality.

Definition 2.7. Assume $p \in S(A)$ and $q \in S(B)$ are $*$-algebraic.

(1) If $A=B$, then we say that $p$ and $q$ are almost $m$-orthogonal if for every $a \in p(\mathfrak{C})$ and $b \in q(\mathfrak{C})$ we have $a \stackrel{\mathrm{m}}{\downarrow} b(A)$.

(2) If $A=B$ then we say that $p$ and $q$ are weakly $m$-orthogonal if $p(x) \cup q(y)$ extends uniquely to a complete type in variables $x, y$ over $A$.

(3) We say that $p$ and $q$ are $m$-orthogonal if any their m-free extensions over a common domain are almost m-orthogonal.

REMARK 2.8. Assume $p, q \in S(A)$ are *-algebraic. Then $p$ and $q$ are almost $m$-orthogonal iff $p(x) \cup q(y)$ has finitely many completions over $A$.

Proof. Let $a \in p(\mathfrak{C})$. For $b \in q(\mathfrak{C})$, the type of $a b$ over $A$ is determined by $\operatorname{Tr}_{A}(b / A a)$. If $p$ and $q$ are almost m-orthogonal, then this trace is always clopen in $q(\mathfrak{C})$, hence by the compactness of $q(\mathfrak{C})$, there are finitely many possibilities for $\operatorname{tp}(a b / A)$.

For the other direction, if $q(\mathfrak{C})$ is covered by finitely many closed disjoint sets of the form $\operatorname{Tr}_{A}(b / A a)$, then it follows that all these sets are clopen, meaning that $a \stackrel{\mathrm{m}}{\downarrow} b(A)$.

Now suppose $p \in S_{I}(A)$ is $*$-algebraic of $\mathcal{M}$-rank 1 . In this case $\operatorname{acl}_{A}$ is a pregeometry on $p(\mathfrak{C})$. However this pregeometry is not homogeneous, meaning for instance that the type of an $\mathrm{acl}_{A}$-independent pair of realizations of $p$ is not uniquely determined. In order to make this geometry homogeneous we must localize it with respect to a flat Morley sequence $J$ in $p$.

The notion of a flat Morley sequence in a type $p$ is defined in [Ne6]. Recall that $J \subseteq p(\mathfrak{C})$ is a flat Morley sequence in $p$ if the following conditions hold.

(1) $J$ is m-independent over $A$ (that is, every finite set $J^{\prime} \subset J$ is mindependent over $A$ ).

(2) $J$ is dense in $p(\mathfrak{C})$.

It is rather easy to see that flat Morley sequences exist. In our case, since $\mathcal{M}(p)>0$, each flat Morley sequence in $p$ is infinite. More importantly, by [Ne6, Lemma 3.1], if $J$ and $J^{\prime}$ are two countably infinite Morley sequences in $p$, then $J$ and $J^{\prime}$ are isomorphic via an elementary mapping over $A$.

Definition 2.9. Assume $p \in S_{I}(A)$ is $*$-algebraic of $\mathcal{M}$-rank 1 .

(1) We say that $p$ is locally modular if for some (equivalently: any) countable flat Morley sequence $J$ in $p$, the localized pregeometry induced on $p(\mathfrak{C})$ by $\operatorname{acl}_{A J}$ is modular. 
(2) We say that $p$ is unidimensional if any two m-free extensions of $p$ are m-non-orthogonal.

(3) We say that $p$ is non-trivial if $\operatorname{acl}_{A}$ is non-trivial on $p(\mathfrak{C})$, that is, there is some finite set $B \subseteq p(\mathfrak{C})$ of size $\geq 3$ which is m-dependent over $A$, but every two-element subset of it is m-independent over $A$.

Notice that any unidimensional type $p$ is non-trivial.

REMARK 2.10. If $T$ is small and $p \in S(A)$ is *-algebraic of $\mathcal{M}$-rank 1 , then for any finite $B, \operatorname{acl}_{A}(B) \cap p(\mathfrak{C})$ is finite.

Proof. The proof is similar to the proof of [Ne4, Lemma 2.1].

Remark 2.10 implies that for countable $B, \operatorname{acl}_{A}(B) \cap p(\mathfrak{C})$ is countable, hence meager in $p(\mathfrak{C})$. The next lemma shows that a non-trivial $*$-algebraic type of $\mathcal{M}$-rank 1 splits into finitely many unidimensional types.

LEMMA 2.11. If $T$ is small and $p \in S(A)$ is non-trivial, *-algebraic, and of $\mathcal{M}$-rank 1 , then for some $E \in \mathrm{FE}(A), E$ splits $p(\mathfrak{C})$ into finitely many pairwise m-orthogonal unidimensional types $p_{0}, \ldots, p_{k}$. More specifically, $\left\{p_{0}, \ldots, p_{k}\right\}=\left\{\operatorname{tp}\left(a / A a^{*}\right): a \in p(\mathfrak{C})\right.$ and $a^{*}$ is the E-class of $\left.a\right\}$ (so each $a^{*}$ is standard).

Proof. This corresponds to a result of Buechler [Bu2] saying that if $T$ is small and $q \in S(\emptyset)$ is an isolated weakly minimal non-trivial type, then for some $E^{\prime} \in \mathrm{FE}(\emptyset)$, any two $E^{\prime}$-equivalent stationarizations of $q$ are nonorthogonal. Here the proof is similar. Let $J$ be a flat Morley sequence in $p$. For any $a \in p(\mathfrak{C}) \backslash \operatorname{acl}_{A}(J)$ let $O(a)$ be the topological closure of $\operatorname{acl}_{A J}(a) \cap$ $p(\mathfrak{C})$. It is easy to see that sets of the form $O(a)$ partition $p(\mathfrak{C})$ into finitely many clopen sets, hence are $E$-classes (on $p(\mathfrak{C}))$ for some $E \in \mathrm{FE}(A)$. Clearly $E$ is good.

Theorem 2.12 ([Ne5, Corollary 3.9]). If $T$ is superstable with few countable models, then any *-algebraic type $p \in S_{I}(A)$ of $\mathcal{M}$-rank 1 is locally modular and non-trivial. Also, if $p$ is unidimensional, then the geometry on $p(\mathfrak{C})$ localized with respect to a countable flat Morley sequence in $p$ is projective over some locally finite field.

Lemma 2.13. Assume $T$ is m-normal and $p \in S_{I}(A)$ is *-algebraic of $\mathcal{M}$-rank 1 . Then $p$ is locally modular and if additionally $p$ is unidimensional, then the localized geometry on $p$ is projective.

Proof. We can assume $A=\emptyset$. Let $J$ be a countable flat Morley sequence in $p$. To prove that $\mathrm{acl}_{J}$ is locally modular it is enough to show that any two lines on a plane in this pregeometry intersect. That is, choose a set $\{a, b, c, d\} \subset p(\mathfrak{C}) \backslash \operatorname{acl}(J)$ of dimension 3 of over $J$ and assume that any 3 of its elements are $\operatorname{acl}_{J}$-independent. We must find an $e \in p(\mathfrak{C})$ with $e \in \operatorname{acl}_{J}(a b) \cap \operatorname{acl}_{J}(c d) \backslash \operatorname{acl}_{J}(\emptyset)$. 
By the finite character of acl, choose a finite $J^{\prime} \subset J$ such that $a b \stackrel{\mathrm{m}}{\chi}$ $c d\left(J^{\prime}\right)$. By m-normality, there is an $e^{\prime} \in \operatorname{acl}_{J^{\prime}}(a b) \cap \operatorname{acl}_{J^{\prime}}(c d)$ with $a b \stackrel{\mathrm{m}}{\downarrow}$ $c d\left(J^{\prime} e\right)$. The Lascar inequalities for $\mathcal{M}$-rank yield $\mathcal{M}\left(e^{\prime} / J^{\prime}\right)=1, e^{\prime} \notin \operatorname{acl}(J)$ and $b$ and $e^{\prime}$ are acl $J^{\prime} a^{-i n t e r d e p e n d e n t . ~}$

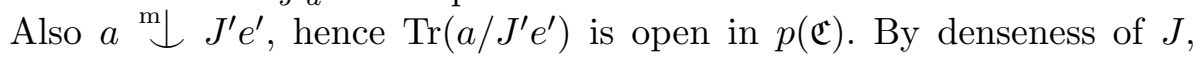
we can choose $a^{\prime} \in J$ with $a \equiv a^{\prime}\left(J^{\prime} e^{\prime}\right)$ and then an $e \in p(\mathfrak{C})$ with $a b \equiv$ $a^{\prime} e\left(J^{\prime} e^{\prime}\right)$. We see that $e$ and $e^{\prime}$ are $\operatorname{acl}_{J^{\prime} a^{\prime}}$-interdependent. Since $e^{\prime} \notin \operatorname{acl}(J)$, also $e \notin \operatorname{acl}(J)$. Clearly, $e \in \operatorname{acl}_{J}(a b) \cap \operatorname{acl}_{J}(c d)$, so we are done.

In order to prove that $\operatorname{acl}_{J}$ is projective on $p$, it is enough to show that any $\operatorname{acl}_{J}$-line has at least 3 points. This may be proved similarly, using unidimensionality of $p$.

The next ingredient we shall need to characterize m-normality is mcoordinatization.

Definition 2.14. (1) We say that $T$ has weak $m$-coordinatization if every *-algebraic type of $\mathcal{M}$-rank $>0$ is m-non-orthogonal to a $*$-algebraic type of $\mathcal{M}$-rank 1 .

(2) We say that $T$ has full $m$-coordinatization if for every $A$ and $a *$ algebraic over $A$ with $\mathcal{M}(a / A)>0$ there is $b \in \operatorname{acl}_{A}(a)$ with $\mathcal{M}(b / A)=1$.

THEOREM 2.15 ([Ne5, Theorem 2.1]). If $T$ is superstable with few countable models, then $T$ has weak m-coordinatization.

LEMMA 2.16. If $T$ is m-normal and $m$-stable, then $T$ has full $m$-coordinatization.

Proof. Assume $a$ is *-algebraic over $A$ and $\mathcal{M}(a / A)>0$. By Corollary $2.5, \mathcal{M}(a / A)$ is finite. Choose a $b *$-algebraic over $A$ with $\mathcal{M}(a / A b)=$ $\mathcal{M}(a / A)-1$. By m-normality, choose $c \in \operatorname{acl}_{A}(a) \cap \operatorname{acl}_{A}(b)$ with $a \stackrel{\text { m }}{\downarrow} b(A c)$. The Lascar inequalities yield $\mathcal{M}(c / A)=1$.

3. m-normality, m-coordinatization and local modularity. In this section we show that for small theories of finite $\mathcal{M}$-rank, m-normality is equivalent to m-coordinatization and local modularity of all *-algebraic types of $\mathcal{M}$-rank 1 . The hard part of the proof is to show that m-coordinatization and the local modularity assumption imply m-normality. First we deal with some special cases.

Remark 3.1. Assume $A \subseteq B$ are finite and $a$ is $*$-algebraic over $A$. If $a \stackrel{\mathrm{m}}{\perp} B(A)$ or $\mathcal{M}(a / B)=0$ then $\operatorname{Tr}_{A}(a / B)$ is locally almost Aa-invariant.

Proof. If $a \stackrel{\mathrm{m} \downarrow}{ } B(A)$ then for some $E \in \mathrm{FE}(A), \operatorname{Tr}_{A}(a / B) \cap[E(x, a)]=$ $\operatorname{Tr}_{A}(a / A) \cap[E(x, a)]$, hence $\operatorname{Tr}_{A}(a / B) \cap[E(x, a)]$ is $A a$-invariant. If $\mathcal{M}(a / B)$ $=0$, then $\operatorname{Tr}_{A}(a / B)$ is finite, hence locally almost $A a$-invariant.

LEMMA 3.2. Assume $T$ is small, m-stable and every *-algebraic type of $\mathcal{M}$-rank 1 is locally modular. 
(1) Assume $A \subseteq B$ are finite, $p \in S(A)$ is $*$-algebraic of $\mathcal{M}$-rank 1 and $a_{1}, a_{2}$ are m-independent realizations of $p$. Then $\operatorname{Tr}_{A}\left(a_{1} a_{2} / B\right)$ is locally almost $A a_{1} a_{2}$-invariant.

(2) Assume $A \subseteq B$ are finite, $a_{1}, a_{2}$ are $*$-algebraic and m-independent over $A$ and $\mathcal{M}\left(a_{1} / A\right)=\mathcal{M}\left(a_{2} / A\right)=1$. Then $\operatorname{Tr}_{A}\left(a_{1} a_{2} / B\right)$ is locally almost $A a_{1} a_{2}$-invariant.

Proof. (1) Let $q=\operatorname{tp}\left(a_{1} a_{2} / A\right)$ and let $X=\operatorname{Tr}_{A}\left(a_{1} a_{2} / B\right)$. Since we are interested in local almost invariance of $X$, we may freely add to $A$ finitely many parameters algebraic over $A$. By Remark 3.1 we are reduced to the case when $\mathcal{M}\left(a_{1} a_{2} / B\right)=1$. Notice that $q(\mathfrak{C})$ is a clopen subset of $p(\mathfrak{C}) \times p(\mathfrak{C})$. So $\mathcal{M}\left(a_{1} a_{2} / B\right)=1$ means just that $X$ is an infinite closed nowhere dense subset of $p(\mathfrak{C}) \times p(\mathfrak{C})$. Let $\pi_{i}: p(\mathfrak{C}) \times p(\mathfrak{C}) \rightarrow p(\mathfrak{C})$ be the projection onto the $i$ th coordinate, $i=1,2$. Notice that $\pi_{i}(X)=\operatorname{Tr}_{A}\left(a_{i} / B\right)$. Let $b$ be the *-algebraic (over $A$ ) name of $X$.

Case 1: For some $i, \pi_{i}(X)$ is finite. Say, this happens for $i=1$. Extending $A$ by an algebraic parameter we may assume that $\pi_{1}(X)=\left\{a_{1}\right\}$. Then $X$ is an open subset of $\left\{a_{1}\right\} \times q(\mathfrak{C})_{a_{1}}$, where $q(\mathfrak{C})_{a_{1}}$ is the vertical section of $q(\mathfrak{C})$ at $a_{1}$.

But $q(\mathfrak{C})_{a_{1}}$ is open in $p(\mathfrak{C})$, hence $X$ is open in $\left\{a_{1}\right\} \times p(\mathfrak{C})$. So for some $E \in \mathrm{FE}(A)$ we have

$$
X \cap\left[E\left(x_{1} x_{2}, a_{1} a_{2}\right)\right]=\left\{a_{1}\right\} \times q(\mathfrak{C})_{a_{1}} \cap\left[E\left(x_{1} x_{2}, a_{1} a_{2}\right)\right] .
$$

The latter set is clearly $A a_{1} a_{2}$-invariant.

Case 2: For $i=1,2, \pi_{i}(X)$ is infinite. Then each $X_{i}=\pi_{i}(X)$ is clopen in $p(\mathfrak{C})$ (as $\mathcal{M}(p)=1$ ) and $X \subseteq X_{1} \times X_{2}$. So $a_{i}{ }^{\stackrel{\mathrm{m}}{\downarrow} b} b(A)$. Since $\mathcal{M}\left(a_{1} a_{2} / B\right)=1$, all vertical and horizontal sections of $X$ are finite. Extending $A$ if necessary, we may assume these sections have size $\leq 1$.

By Lemma 2.6 we find a finite set $C \subseteq p(\mathfrak{C})$ with $b \in \operatorname{acl}_{A}(C)$. Without loss of generality, $a_{1} a_{2}{ }^{\mathrm{m}} \mathfrak{L} C(A b)$. So $a_{i}{ }^{\mathrm{m}} \mathcal{L} C(A)$. Let $J$ be a countable flat Morley sequence in $p$. We can assume that $J \cup\left\{a_{1}, C\right\}$ is m-independent over $A$.

We have $\mathcal{M}\left(a_{1} a_{2} / A\right)=2$ and $\mathcal{M}\left(a_{1} a_{2} / A C\right)=1$. By modularity of $\operatorname{acl}_{A J}$ on $p(\mathfrak{C})$, there is an $e \in p(\mathfrak{C})$ with

$$
e \in \operatorname{acl}_{A J}\left(a_{1} a_{2}\right) \cap \operatorname{acl}_{A J}(C) \backslash \operatorname{acl}_{A}(J) .
$$

In particular, for some finite $J^{\prime} \subseteq J$ we have $e \in \operatorname{acl}_{A J^{\prime}}\left(a_{1} a_{2}\right) \cap \operatorname{acl}_{A J^{\prime}}(C)$,

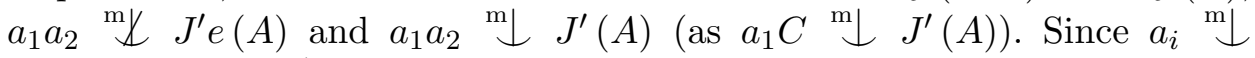

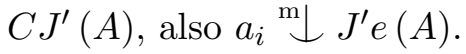

Let $Y=\operatorname{Tr}_{A}\left(a_{1} a_{2} / A J^{\prime} e\right)$. So $\pi_{i}(Y)$ is open in $p(\mathfrak{C})$ for $i=1,2$. Since $\mathcal{M}\left(a_{1} a_{2} / A J^{\prime} e\right)=1$, all sections of $Y$ are finite, so we may assume they have 
size at most 1 . In particular,

$$
X_{a_{1}}=Y_{a_{1}} .
$$

Clearly, (a) also holds for any $a_{1}^{\prime} \equiv a_{1}\left(A b J^{\prime} e\right)$. But $a_{1} \stackrel{\mathrm{m}}{\perp} b J^{\prime} e(A)$, so $\operatorname{Tr}_{A}\left(a_{1} / A b J^{\prime} e\right)$ is open in $p(\mathfrak{C})$. This means that for some $E\left(x_{1} x_{2}, x_{1}^{\prime} x_{2}^{\prime}\right) \in$ $\mathrm{FE}(A), X \cap\left[E\left(x_{1} x_{2}, a_{1} a_{2}\right)\right]=Y \cap\left[E\left(x_{1} x_{2}, a_{1} a_{2}\right)\right]$.

Let $d$ be the name of $X \cap\left[E\left(x_{1} x_{2}, a_{1} a_{2}\right)\right]$. We see that $d \in \operatorname{acl}_{A}(b) \cap$

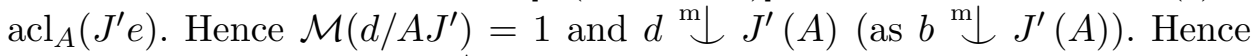
$\mathcal{M}(d / A)=1$. Also, $a_{1} a_{2}{ }^{\mathrm{m}} \mathcal{L} d(A)$, hence $d \in \operatorname{acl}_{A}\left(a_{1} a_{2}\right)$. We have shown that $X$ is locally almost $A a_{1} a_{2}$-invariant, proving (1).

(2) Let $p_{i}=\operatorname{tp}\left(a_{i} / A\right)$. As in the proof of (1) we can assume $B=A b$, where $b$ is the name of $\operatorname{Tr}_{A}\left(a_{1} a_{2} / B\right)$. Also, neglecting some easy cases we can assume that $a_{1}{ }^{\mathrm{m}} \mathfrak{L} b(A), a_{2}{ }^{\mathrm{m}} \mathfrak{L} b(A)$ and $\mathcal{M}\left(a_{1} a_{2} / A b\right)=1$. This means that $a_{1}{ }^{\mathrm{m}} \mathcal{L} a_{2}(A b)$, hence $p_{1}$ and $p_{2}$ are m-non-orthogonal. We can assume that all sections of $\operatorname{Tr}_{A}\left(a_{1} a_{2} / B\right) \subseteq p_{1}(\mathfrak{C}) \times p_{2}(\mathfrak{C})$ have size $\leq 1$.

Choose $b^{\prime} a_{1}^{\prime} a_{2}^{\prime} \equiv b a_{1} a_{2}(A)$ with $b^{\prime} a_{1}^{\prime} a_{2}^{\prime}{ }^{\mathrm{m} \downarrow} b a_{1} a_{2}(A)$ and let $U=$ $\operatorname{Tr}_{A}\left(a_{1}^{\prime} a_{2}^{\prime} / A b^{\prime}\right), U_{i}=\operatorname{Tr}_{A}\left(a_{i}^{\prime} / A b^{\prime}\right), i=1,2$. We see that $U_{i} \subseteq p_{i}(\mathfrak{C}), i=1,2$, are clopen and $U$ is the graph of an $A b^{\prime}$-definable bijection $h: U_{1} \rightarrow U_{2}$.

We can assume that $a_{1} \in U_{1}$. Let $X=\operatorname{Tr}_{A}\left(a_{1} a_{2} / A b b^{\prime}\right) \subseteq U_{1} \times p_{2}(\mathfrak{C})$ and let $Y=\operatorname{Tr}_{A}\left(h\left(a_{1}\right) a_{2} / A b b^{\prime}\right)=(h \times \mathrm{id})(X)$. Since $a_{1} a_{2}{ }^{\mathrm{m} \downarrow} b^{\prime}(A b)$, $X$ is clopen in $\operatorname{Tr}_{A}\left(a_{1} a_{2} / B\right)$. By (1), $Y$ is locally almost $A h\left(a_{1}\right) a_{2}$-invariant, hence locally almost $A a_{1} a_{2} b^{\prime}$-invariant. So $X$ is also locally almost $A a_{1} a_{2} b^{\prime}$ invariant. Let $b^{*}$ be the name of a clopen neighbourhood of $a_{1} a_{2}$ in $X$, which is algebraic over $A a_{1} a_{2} b^{\prime}$. Also, $b^{*} \in \operatorname{acl}_{A}(b)$. As $b^{\prime}{ }^{\mathrm{m}} \downarrow b\left(A a_{1} a_{2}\right)$, we conclude that $b^{*} \in \operatorname{acl}\left(A a_{1} a_{2}\right)$. So $\operatorname{Tr}_{A}\left(a_{1} a_{2} / B\right)$ is locally almost $A a_{1} a_{2}$-invariant.

The next theorem is the main result of this section.

Theorem 3.3. Assume $T$ is small and the $\mathcal{M}$-rank of any *-algebraic type in $T$ is finite. Then the following conditions are equivalent.

(1) $T$ is m-normal.

(2) Every *-algebraic $\mathcal{M}$-rank 1 type in $T$ is locally modular and $T$ has full m-coordinatization.

(3) Every *-algebraic $\mathcal{M}$-rank 1 type in $T$ is locally modular and $T$ has weak m-coordinatization.

Proof. By the results from Section 2 we see that $(1) \Rightarrow(2) \Rightarrow(3)$. So we must prove $(3) \Rightarrow(1)$. Assuming (3) we will prove that condition (2) of Theorem 2.3 holds. That is, we shall prove that

(*) if $A \subseteq B$ are finite and $a$ is $*$-algebraic over $A$, then $\operatorname{Tr}_{A}(a / B)$ is locally almost $A a$-invariant. 
We prove $(*)$ by induction on $n=\mathcal{M}(a / A)$. For $n=0,1,(*)$ follows from Remark 3.1.

STEP 1: $n=2$. We can assume that $B=A b$, where $b$ is the name of $\operatorname{Tr}_{A}(a / B)$. Let $r_{b}=\operatorname{tp}(a / A b)$. By Remark 3.1 we can assume that $\mathcal{M}(a / B)$ $=1$. By weak m-coordinatization there is a finite set $C$ and a tuple $c *$ algebraic over $C$ such that $c \in \operatorname{acl}_{A C}(a), \mathcal{M}(c / A C)=1$ and $a \stackrel{\mathrm{m}}{\downarrow} C(A)$. Without loss of generality, $c \in \operatorname{dcl}_{A C}(a)$ and $a b \stackrel{\mathrm{m}}{\downarrow} C(A)$.

So $a \stackrel{\mathrm{m} \downarrow}{ } C(A b)$ and $\mathcal{M}(a / A C b)=\mathcal{M}(a / A b)=1$. If $b^{\prime}$ is the name of any clopen neighbourhood of $a$ in $\operatorname{Tr}_{A}(a / A b)$, then $b^{\prime} \in \operatorname{acl}_{A}(b)$, so also $a b^{\prime} \stackrel{\mathrm{m}}{\downarrow} C(A)$. Hence if we prove that $b^{\prime} \in \operatorname{acl}_{A C}(a)$, then $b^{\prime} \stackrel{\mathrm{m}}{\downarrow} C(A a)$ gives $b^{\prime} \in \operatorname{acl}_{A}(a)$. Therefore it is enough to prove that $\operatorname{Tr}_{A}(a / A b C)=$ $\operatorname{Tr}_{A C}(a / A b C)$ is locally almost $A C a$-invariant. So we can assume that $C=\emptyset$.

Let $p=\operatorname{tp}(a / A)$ and $q=\operatorname{tp}(c / A)$. Since $c \in \operatorname{dcl}_{A}(a)$, there is a tuple of $A$-definable functions $f: p(\mathfrak{C}) \rightarrow q(\mathfrak{C})$ with $f(a)=c$. This gives a typedefinable equivalence relation $E_{f}$ on $p$ defined by

$$
a_{0} E_{f} a_{1} \Leftrightarrow f\left(a_{0}\right)=f\left(a_{1}\right) .
$$

Let $p_{c}=\operatorname{tp}(a / A c)$. So $\mathcal{M}\left(p_{c}\right)=1$ and $p_{c}(\mathfrak{C})$ is the $E_{f}$-class of $a$. For any $c^{\prime} \in q(\mathfrak{C})$ let $p_{c^{\prime}}$ be the $A$-conjugate of $p_{c}$.

If $\operatorname{Tr}_{A}(c / A b)$ is finite, then we prove that $\operatorname{Tr}_{A}(a / A b)$ is locally almost $A a$-invariant as in the proof of Lemma 3.2, case 1.

So we may assume that $\operatorname{Tr}_{A}(c / A b)$ is clopen in $q(\mathfrak{C})$ and that $p_{c}(\mathfrak{C}) \cap$ $\operatorname{Tr}_{A}(a / A b)=\operatorname{Tr}_{A}(a / A b c)$ is finite. As in the proof of Lemma 3.2 we may assume that $p_{c}(\mathfrak{C}) \cap \operatorname{Tr}_{A}(a / A b)$ has size 1 and $\operatorname{Tr}_{A}(c / A b)=q(\mathfrak{C})$. So $a \in$ $\operatorname{dcl}_{A b}(c)$.

Now we can think of the type $p$ and the function $f: p(\mathfrak{C}) \rightarrow q(\mathfrak{C})$ as a "cover" of $q$, with fibers $p_{\mathcal{c}^{\prime}}(\mathfrak{C}), c^{\prime} \in q(\mathfrak{C})$, and a transversal section $\operatorname{Tr}_{A}(a / A b)$. Let $g_{b}: q(\mathfrak{C}) \rightarrow p(\mathfrak{C})$ be the $A b$-definable function mapping $c$ to $a$. So $g_{b}$ is a bijection between $q(\mathfrak{C})$ and $\operatorname{Tr}_{A}(a / A b)$.

We will reduce proving that $\operatorname{Tr}_{A}(a / A b)$ is locally almost $A a$-invariant to the situation considered in Lemma 3.2. The main point of this reduction is that in our case all the types $p_{c^{\prime}}, c^{\prime} \in q(\mathfrak{C})$, are m-non-orthogonal to $q$ (although this is not clear at this point yet).

Choose $b^{*} \equiv b(A)$ with $b^{*} \stackrel{\mathrm{m}}{\downarrow} a b(A)$. Let $r_{b^{*}}, g_{b^{*}}$ be the $A$-conjugates of $r_{b}, g_{b}$ respectively. Notice that $r_{b^{*}}=\operatorname{tp}\left(g_{b^{*}}(c) / A b^{*}\right)$. By Lemma 2.6 choose a finite set $D$ of realizations of $r_{b^{*}}$ such that $\operatorname{Tr}_{A}\left(r_{b^{*}}\right)$ is $A D$-invariant. We can assume that $c \stackrel{\mathrm{m} \downarrow}{\perp}(A)$. Since $\operatorname{Tr}_{A}\left(r_{b^{*}}\right)=\operatorname{Tr}_{A}\left(g_{b^{*}}(c) / A b^{*}\right)$, we see that $g_{b^{*}}$ is $A D$-definable, hence $g_{b^{*}}(c) \in \operatorname{acl}_{A}(D c)$.

Now we can choose $D \subset r_{b^{*}}(\mathfrak{C})$ as in the last paragraph of minimal size such that $c \stackrel{\mathrm{m} \downarrow}{\perp} D(A)$ and $g_{b^{*}}(c) \in \operatorname{acl}_{A}(D c)$. Say, $D=\left\{d_{0}, \ldots, d_{k}\right\}=d_{\leq k}$. Let $c_{k}=f\left(d_{k}\right)$. By the minimality of $D$ there are two cases: either $g_{b^{*}}(c) \in$ $\operatorname{acl}_{A}\left(d_{<k} c_{k} c\right) \backslash \operatorname{acl}_{A}\left(d_{<k} c\right)$ or $g_{b^{*}}(c) \in \operatorname{acl}_{A}(D c) \backslash \operatorname{acl}_{A}\left(d_{<k} c_{k} c\right)$. 
CASE 1.1: $g_{b^{*}}(c) \notin \operatorname{acl}_{A}\left(d_{<k} c_{k} c\right)$. Then $d_{k} \notin \operatorname{acl}_{A}\left(d_{<k} c_{k} c\right)$. Let $D^{\prime}=$ $d_{<k} c_{k}$. Since $d_{k}$ and $g_{b^{*}}(c)$ are interalgebraic over $A D^{\prime} c$, we can find an $e \in \operatorname{acl}(A)$ such that they are interdefinable over $A D^{\prime} c e$.

Let $s(x, y, z)=\operatorname{tp}\left(c d_{k} g_{b^{*}}(c) / A D^{\prime} e\right)$. Then $\mathcal{M}(s)=2, s(\mathfrak{C}) \subseteq q(\mathfrak{C}) \times$ $p_{c_{k}}(\mathfrak{C}) \times p(\mathfrak{C})$.

Let $\pi_{i j}, \pi_{i}, i=1,2,3$, be the projections from $q(\mathfrak{C}) \times p_{c_{k}}(\mathfrak{C}) \times p(\mathfrak{C})$ to suitable subproducts or coordinates.

Since $\mathcal{M}\left(g_{b^{*}}(c) / A D^{\prime} e\right)=2=\mathcal{M}(p)$, we see that all the sets $U_{i}=$ $\pi_{i}(s(\mathfrak{C})), i=1,2,3$, are clopen in the respective axes and $U_{12}=\pi_{12}(s(\mathfrak{C}))$ is clopen in $q(\mathfrak{C}) \times p_{c_{k}}(\mathfrak{C})$. Also, $s(\mathfrak{C})$ is the graph of an $A D^{\prime} e$-definable bijection $h(x, y): U_{12} \rightarrow U_{3}$.

Now we can assume that $D \stackrel{\mathrm{m}}{\Perp} a b(A)$ and $a \in U_{3}$. Then also $a \stackrel{\mathrm{m}}{\Perp}$ $D^{\prime} e(A b)$ and $Y=\operatorname{Tr}_{A}\left(a / A b D^{\prime} e\right)$ is clopen in $\operatorname{Tr}_{A}(a / A b)$ and contained in $U_{3}$.

Let $Z=h^{-1}[Y]=\operatorname{Tr}_{A c_{k}}\left(h^{-1}(a) / A b D^{\prime} e\right) \subseteq q(\mathfrak{C}) \times p_{c_{k}}(\mathfrak{C})$. By Lemma 3.2, $Z$ is locally almost $A c_{k} h^{-1}(a)$-invariant, hence locally almost $A D a$-invariant. It follows that also $Y$ is locally almost $A D a$-invariant. Choose a name $b^{+}$of a clopen neighbourhood of $a$ in $Y$ with $b^{+} \in \operatorname{acl}_{A}(D a)$. Also, $b^{+} \in \operatorname{acl}_{A}(b)$. Since $D a \stackrel{\mathrm{m} \downarrow}{\perp} b(A a)$, we deduce that $b^{+} \in \operatorname{acl}_{A}(a)$, so $Y$ and $\operatorname{Tr}_{A}(a / A b)$ are locally almost $A a$-invariant.

CASE 1.2: $g_{b^{*}}(c) \in \operatorname{acl}_{A}\left(d_{<k} c_{k} c\right) \backslash \operatorname{acl}_{A}\left(d_{<k} c\right)$. In this case proceed as in Case 1.1, this time however considering $s=\operatorname{tp}\left(c c_{k} g_{b^{*}}(c) / A d_{<k} e\right)$ for suitable $e \in \operatorname{acl}(A)$, with $s(\mathfrak{C}) \subseteq q(\mathfrak{C}) \times q(\mathfrak{C}) \times p(\mathfrak{C})$

This finishes the proof of $(*)$ in the case where $n=2$.

Step 2: Suppose $n>2$ and $(*)$ is true for every $n^{\prime}<\alpha$. Again we may assume $B=A b$, where $b$ is the name of $\operatorname{Tr}_{A}(a / B)$. By weak mcoordinatization, for some finite $C$ with $a b^{\mathrm{m}} \mathcal{L} C(A)$ there is $c_{1} \in \operatorname{acl}_{A C}(a)$ *-algebraic over $C$ with $\mathcal{M}\left(c_{1} / A C\right)=1$. As in Step 1 we can assume that $C=\emptyset$ and $c_{1} \in \operatorname{dcl}_{A}(a)$. Now $\mathcal{M}\left(a / A c_{1}\right)=n-1$. Choose $c_{2} *-$ algebraic over $A$ with $\mathcal{M}\left(a / A c_{1} c_{2}\right)=n-2$. Again we may assume that $c_{2}$ is the name of $\operatorname{Tr}_{A}\left(a / A c_{1} c_{2}\right)=\operatorname{Tr}_{A c_{1}}\left(a / A c_{1} c_{2}\right)$. By the induction hypothesis, $\operatorname{Tr}_{A c_{1}}\left(a / A c_{1} c_{2}\right)$ is locally almost $A c_{1} a$-invariant, meaning that we can take $c_{2} \in \operatorname{acl}_{A c_{1}}(a)$, which implies $\mathcal{M}\left(c_{2} / A c_{1}\right)=1$. Again without loss of generality, $c_{2} \in \operatorname{dcl}_{A c_{1}}(a)$.

Continuing in this manner we get a tower of elements $c_{1}, \ldots, c_{n-1} \in$ $\operatorname{dcl}_{A}(a)$ with $\mathcal{M}\left(c_{i} / A c_{<i}\right)=1$ and $\mathcal{M}\left(a / A c_{<n}\right)=1$. Let $d_{i}=c_{\leq i}$. So $\mathcal{M}\left(d_{i} / A\right)=i$ for $i=1, \ldots, n-1$. We can assume that $0<\mathcal{M}(a / A b)<n$.

CAsE 2.1: $\mathcal{M}\left(d_{n-1} / A b\right)<n-1$. By the induction hypothesis, we know that $\operatorname{Tr}_{A}\left(d_{n-1} / A b\right)$ is locally almost $A d_{n-1}$-invariant. Extending $A$ by an $e \in \operatorname{acl}(A)$ (by Lemma 2.2) we may assume that $\operatorname{Tr}_{A}\left(d_{n-1} / A b\right)$ is $A d_{n-1^{-}}$ invariant. Let $c$ be the name of $\operatorname{Tr}_{A}\left(d_{n-1} / A b\right)$. Then $\mathcal{M}(a / A c)<n$ (as 
$\left.d_{n-1} \stackrel{\mathrm{m} \not}{\chi} c(A)\right)$, so $\operatorname{Tr}_{A c}(a / A c b)$ is locally almost $A c a$-invariant. But $\operatorname{Tr}_{A c}(a / A c b)=\operatorname{Tr}_{A}(a / A c b)=\operatorname{Tr}_{A}(a / A b)$, as $c \in \operatorname{dcl}(A b) \cap \operatorname{dcl}(A a)$. So $\operatorname{Tr}_{A}(a / A b)$ is locally $A a$-invariant.

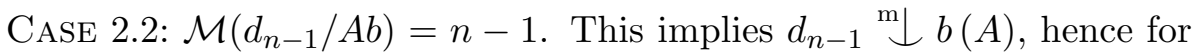
each $i, d_{i}{ }^{\mathrm{m}} \downarrow b(A)$. We have $\mathcal{M}\left(a / A d_{1} b\right)>0$. Indeed, otherwise $\mathcal{M}\left(d_{2} / A d_{1} b\right)$ $=0$, so $\mathcal{M}\left(d_{2} / A b\right)=\mathcal{M}\left(d_{1} / A b\right)=1<\mathcal{M}\left(d_{2} / A\right)$, contradicting $d_{2}{ }^{\mathrm{m}} \downarrow$

Also, $\mathcal{M}\left(a / A d_{1}\right)=n-1$ and $\mathcal{M}\left(a / A d_{1} b\right)=l<n-1$. So by the induction hypothesis, $\operatorname{Tr}_{A}\left(a / A d_{1} b\right)$ is locally almost $A d_{1} a$-invariant. By Lemma 2.2 we may assume it is $A d_{1} a$-invariant, hence also $A a$-invariant.

Let $b_{n-l} \in \operatorname{dcl}_{A}(a)$ be the name of $\operatorname{Tr}_{A}\left(a / A d_{1} b\right)$. So $\mathcal{M}\left(a / A b_{n-l}\right)$ $=\mathcal{M}\left(a / A d_{1} b\right)=l$ and $0<l<n-1$, hence $\mathcal{M}\left(b_{n-l} / A\right)=n-l$ and $a \stackrel{\mathrm{m}}{\perp} b\left(A b_{n-l}\right)$. As above we can find elements $b_{n-l+1}, \ldots, b_{n-1} \in \operatorname{dcl}_{A}(a)$ such that $b_{i} \in \operatorname{dcl}_{A}\left(b_{i+1}\right)$ and $\mathcal{M}\left(b_{i} / A\right)=i$ for all $i$. But $b_{n-l}{ }^{\mathrm{m}} \mathcal{L} b(A)$, so

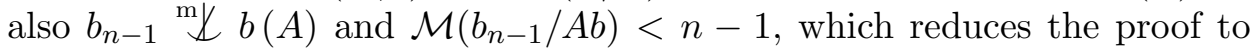
Case 2.1.

Comparing Theorem 3.3 with the characterization of 1-based theories among superstable theories of finite rank from [Bu1] we see that in [Bu1] the (full) coordinatization follows from 1-basedness, but the local modularity assumption alone implies 1-basedness (i.e. we do not need "weak coordinatization" as in condition (2) of Theorem 3.3). This is because within the theory of forking, weak coordinatization holds for every superstable theory of finite rank, meaning that any non-algebraic type in such a theory is non-orthogonal to a regular type of $U$-rank 1 . As the reader sees from Theorem 2.15, in order to prove that a superstable theory has weak mcoordinatization we needed the few models assumption. It is not clear if this assumption may be weakened to smallness.

By Theorems 2.12 and 2.15 we can draw the following corollary. Notice that (2) in this corollary improves [Ne5, Theorem 2.1].

Corollary 3.4. If $T$ is superstable with few countable models, then

(1) $T$ is m-normal and

(2) $T$ has full m-coordinatization.

I think that thus far Corollary 3.4, together with the results from the next section, is the main justification for introducing the notion of m-normal theory.

4. *-algebraic groups in m-normal theories. Recall from [Ne5] that a $*$-algebraic group $G$ is a type-definable (over some finite set) group of uniformly $*$-finite tuples. That is, there is a finite set $A$, a set $X$ of standard elements, type-definable over $A$, and a tuple of $A$-definable functions $f$ on $X$ such that $G=\{f(a): a \in X\}$ and the group operation is type-definable 
over $A$. When elements of $G$ are *-algebraic over $A$, we call $G$ a *-algebraic group (over $A$ ).

The most natural example of a $*$-algebraic group arises as follows. Suppose $H$ is a (standard) group in $T$, type-definable over $A$, and $H_{n}, n<\omega$, is a family of relatively $A$-definable subgroups of finite index in $H$. Let $H_{\infty}=\bigcap_{n} H_{n}$. Then any element $a / H_{\infty}$ of $H / H_{\infty}$ is interdefinable over $A$ with the $*$-algebraic tuple $\left\langle a / H_{n}, n<\omega\right\rangle$. Hence if $H_{\infty}$ is normal in $H$, then the quotient group $H / H_{\infty}$ is a $*$-algebraic group. In particular, if $T$ is stable then the group $H / H^{0}$ is $*$-algebraic $\left(H^{0}\right.$ is the connected component of $H$ ). In fact, we can regard $H / H^{0}$ as the group of generic types of $H$.

In this section we assume $G$ is a *-algebraic group over $\emptyset$. Again, there is a natural compact 0-dimensional topology on $G$ induced by the base of open sets of the form $\left[b_{J}\right]=\left\{a_{I} \in G: a_{J}=b_{J}\right\}$ (here $I$ is the index set for the *-algebraic tuples in $G, J \subseteq I$ is finite and $b_{J}$ is any $J$-tuple). Hence $G$ is a topological, profinite group and has a basis of neighbourhoods of 1 consisting of 0-definable normal subgroups $G_{n}, n<\omega$, of finite index. We assume that $G_{n+1} \subseteq G_{n}$ for all $n$.

We call a type $p \in S(A)$ of elements of $G$ m-generic (over $A$ ) if $p(\mathfrak{C}$ ) is open in $G$. We say that $a \in G$ is $m$-generic over $A$ if $\operatorname{tp}(a / A)$ is mgeneric. It is not hard to see that if $p, q$ are two m-generic types in $G$, then $\mathcal{M}(p)=\mathcal{M}(q)$.

Indeed, we can assume $p$ and $q$ have a common domain $A$. Choose $a \in p(\mathfrak{C})$ and $b \in q(\mathfrak{C})$ with $a \stackrel{\mathrm{m} \downarrow}{\downarrow} b(A)$. Let $c=a^{-1} b$. Then $c \stackrel{\mathrm{m}}{\downarrow} a(A), c \stackrel{\mathrm{m}}{\downarrow} b(A)$ and the right translation by $c$ carries $\operatorname{Tr}_{A}(a / A c)$ to $\operatorname{Tr}_{A}(b / A c)$. This shows that

$$
\mathcal{M}(a / A)=\mathcal{M}(a / A c)=\mathcal{M}(b / A c)=\mathcal{M}(b / A) .
$$

So we define the $\mathcal{M}$-rank of $G, \mathcal{M}(G)$, as $\mathcal{M}(p)$ for any m-generic type $p$. The results of this section correspond to the classical results of Hrushovski and Pillay on 1-based stable groups $[\mathrm{HP}]$. The proofs are often similar to the ones in $[\mathrm{Pi}]$.

While $\mathcal{M}$-rank turns out to be quite a good relative measure of the size of a $*$-algebraic type ("relative", since it really measures the sizes of $*$-algebraic types by comparing them to each other), it is useless when the theory is not m-stable. In that case we can use another, "absolute" measure of the size of a *-algebraic type (used in [Ne1, Ne2]), which we now recall.

Assume $p$ is a complete type of elements of $G$, over a finite domain. We associate with $p$ a function $f(p): \omega \rightarrow \omega$ in the following way. Suppose $X$ is a $G_{n-1}$-coset meeting $p(\mathfrak{C})$. We define $f(p)(n)$ as the number of $G_{n^{-}}$ cosets contained in $X$ and meeting $p(\mathfrak{C})$. We stipulate $G_{-1}=G$, and $f(a / A)$ abbreviates $f(\operatorname{tp}(a / A))$.

Similarly to $\mathcal{M}$-rank, this notion is also related to $m$-independence, as the next lemma shows. For $f, g \in \omega^{\omega}, f={ }^{*} g$ means that $f(n)=g(n)$ for almost 
all $n$. Similarly we define the partial order $\leq^{*}$ on $\omega^{\omega} . \mathbf{1}$ denotes the function constantly equal to 1 . Also we define $f_{\max } \in \omega^{\omega}$ by $f_{\max }(n)=\left[G_{n}: G_{n-1}\right]$.

Lemma 4.1. Assume $a \in G$ and $A \subseteq B$ are finite.

(1) $a \stackrel{\mathrm{m}}{\downarrow} B(A) \Leftrightarrow f(a / A)=^{*} f(a / B)$.

(2) $\mathbf{1} \leq f(a / A) \leq f_{\max }$ and $f(a / A)={ }^{*} f_{\max }$ iff $a$ is m-generic over $A$.

(3) $f(a / A)={ }^{*} \mathbf{1} \Leftrightarrow \mathcal{M}(a / A)=0$.

Proof. Easy.

$f(p)$ is an "absolute" measure of $p$ (relative to the choice of $G_{n}$ 's), because in its definition we do not refer to $f(q)$ for any extension $q$ of $p$. Unfortunately this measure does not behave as well as $\mathcal{M}$-rank. We do not have Lascar-style inequalities for $f(p)$.

For $X \subseteq G$ we define the (left) stabilizer of $X$ in $G$ to be the set $\operatorname{Stab}(X)=\{a \in G: a \cdot X=X\}$. So $\operatorname{Stab}(X)$ is a subgroup of $G$.

REMARK 4.2. If $X \subseteq G$ is closed and A-invariant (equivalently: typedefinable over $A)$, then $\operatorname{Stab}(X)$ is also closed and A-invariant.

Proof. For any $n$ let $B_{n}(X)$ be the union of $G_{n}$-cosets meeting $X$. So $a \in \operatorname{Stab}(X)$ iff $a \cdot B_{n}(X)=B_{n}(X)$ for every $n$. Hence $\operatorname{Stab}(X)=$ $\bigcap_{n} \operatorname{Stab}\left(B_{n}(X)\right)$. Every $\operatorname{Stab}\left(B_{n}(X)\right)$ is a clopen $A$-definable subgroup of $G$, so we are done.

TheOREM 4.3. Assume $T$ is small and $m$-normal, $a \in G, A$ is finite and $p=\operatorname{tp}(a / A)$. Then there is a relatively clopen subset $X$ of $p(G)$ which is a (right) coset of $\operatorname{Stab}(X)$. In particular, $\mathcal{M}(\operatorname{Stab}(X))=\mathcal{M}(p)$ and $p(\mathfrak{C})$ is a union of finitely many cosets of type-definable subgroups of $G$.

Proof. We can assume that $A=\emptyset$. Since for any $e \in \operatorname{acl}(\emptyset)$, any completion of $p$ over $e$ is an m-free extension of $p$, we will be freely adding finitely many elements of $\operatorname{acl}(\emptyset)$ to the signature.

Choose $b \in G$ m-generic over $a$ and let $c=b \cdot a$. Hence $c$ is also m-generic over $a$. Since $\operatorname{Tr}(b / a)$ is clopen in $G$, its name is algebraic. So adding this name to the signature we may assume that all the elements of $p(\mathfrak{C})$ have the same type over $b$. It follows that all the elements of $b \cdot p(\mathfrak{C})$ have the same type over $b$, i.e. $\operatorname{Tr}(c / b)=b \cdot p(\mathfrak{C})$, hence

$$
f(c / b)=f(p) \text {. }
$$

The idea of the proof consists in the following. $b$ determines a function $f_{b}: p(\mathfrak{C}) \rightarrow G$ given by $f_{b}(x)=b \cdot x$. We identify $f_{b}$ with the $*$-finite *-algebraic tuple naming its graph. For $b^{\prime} \equiv b$ let $f_{b^{\prime}}: p(\mathfrak{C}) \rightarrow G$ be the conjugate of $f_{b}$. By m-normality, a "germ" $g_{b}$ of $f_{b}$ at $a$ is algebraic over $(a, c) \quad\left(g_{b}\right.$ is the restriction of $f_{b}$ to some open neighbourhood $Y$ of $a$ in $p(\mathfrak{C}))$. Now using some trace calculations we find a type $q$ extending $\operatorname{tp}(b)$ such that for $b^{\prime}, b^{\prime \prime} \in q(\mathfrak{C}), g_{b^{\prime}}$ and $g_{b^{\prime \prime}}$ have the same range and $f(q)=f(p)$. 
So $q(\mathfrak{C}) \cdot q(\mathfrak{C})^{-1} \subseteq \operatorname{Stab}(Y)$. It follows that $\operatorname{Stab}(Y)$ is large enough, i.e. $f(\operatorname{Stab}(Y))=f(p)$.

The details are as follows. We have $a^{\mathrm{m}} \downarrow c$ and $b=c \cdot a^{-1}$, hence

$$
f(b / c)=f\left(a^{-1} / c\right)=f(a / c)={ }^{*} f(a / \emptyset)=f(p) .
$$

By m-normality, choose $d \in \operatorname{acl}(c) \cap \operatorname{acl}(b)$ with $c \stackrel{\mathrm{m}}{\downarrow} b(d)$. Without loss of generality, $\operatorname{Tr}(c / d) \subseteq \operatorname{Tr}(c / b)$. Since $\operatorname{Tr}(c / b d)$ is clopen in both $\operatorname{Tr}(c / b)=$ $b \cdot p(\mathfrak{C})$ and $\operatorname{Tr}(c / d)$, for some clopen $V, U \subseteq G$ we have

$$
\operatorname{Tr}(c / b d)=b \cdot p(\mathfrak{C}) \cap V=\operatorname{Tr}(c / d) \cap U .
$$

Let $d_{1}$ be the name of $\operatorname{Tr}(c / b d)$ and $d_{2}$ the name of $W=b^{-1} \cdot V$. We see that $d_{1} \in \operatorname{acl}(c) \cap \operatorname{dcl}(b d), d_{2} \in \operatorname{acl}(\emptyset)$ and $\operatorname{Tr}(c / b d)=\operatorname{Tr}\left(c / d_{1}\right)=\operatorname{Tr}\left(c / b d_{1}\right)$.

Let $q=\operatorname{tp}\left(b / c d_{1} d_{2}\right)$. Clearly, $f(q)={ }^{*} f(b / c)={ }^{*} f(p)$ and for every $b^{\prime} \in q(\mathfrak{C})$,

$$
\operatorname{Tr}\left(c / b^{\prime} d_{1}\right)=\operatorname{Tr}\left(c / d_{1}\right)=b \cdot p(\mathfrak{C}) \cap V .
$$

On the other hand, $\operatorname{Tr}\left(c / b^{\prime} d_{1}\right)=b^{\prime} \cdot p(\mathfrak{C}) \cap V$. Hence $b \cdot p(\mathfrak{C}) \cap V=b^{\prime} \cdot p(\mathfrak{C}) \cap V$ and $b^{-1} \cdot V=\left(b^{\prime}\right)^{-1} \cdot V=W$. Therefore

$$
\begin{aligned}
p(\mathfrak{C}) \cap W & =b^{-1}\left(b^{\prime} \cdot p(\mathfrak{C}) \cap V\right)=b^{-1} \cdot b^{\prime} \cdot p(\mathfrak{C}) \cap b^{-1} \cdot V \\
& =b^{-1} \cdot b^{\prime} \cdot p(\mathfrak{C}) \cap W=b^{-1} \cdot b^{\prime} \cdot p(\mathfrak{C}) \cap b^{-1} \cdot b^{\prime} \cdot W \\
& =\left(b^{-1} \cdot b^{\prime}\right)(p(\mathfrak{C}) \cap W) .
\end{aligned}
$$

Let $Y=p(\mathfrak{C}) \cap W$, a clopen subset of $p(\mathfrak{C})$. Clearly, $\operatorname{Stab}(Y)$ is a closed, $d_{2}$-invariant subgroup of $G$. Also, for any complete type $s$ of elements of $\operatorname{Stab}(Y), f(s) \leq^{*} f(p)$ (as $p(\mathfrak{C}) \cap W$ is a union of right cosets of $\left.\operatorname{Stab}(Y)\right)$.

On the other hand, by $(\mathrm{c}), q^{-1}(\mathfrak{C}) \cdot q(\mathfrak{C}) \subseteq \operatorname{Stab}(Y)$, so for any m-generic type $s$ of $\operatorname{Stab}(Y), f(s){ }^{*} \geq f(q)={ }^{*} f(p)$, hence

$$
f(s)={ }^{*} f(p) .
$$

Now, $Y$ is a union of right cosets of $\operatorname{Stab}(Y)$, and by $(d)$ these cosets have non-empty interior in $Y$, hence are open in $Y$. Therefore $Y$ is a finite union of them. Let $X$ be the coset of $\operatorname{Stab}(Y)$ containing $a$. Clearly, $\operatorname{Stab}(Y)=$ $\operatorname{Stab}(X)$ and $X$ is clopen in $p(\mathfrak{C})$.

TheOREM 4.4. Assume $T$ is small and $m$-normal, $A$ is finite and $H$ is an A-type-definable subgroup of $G$. Then $H$ contains a closed subgroup $H^{\prime}$ of finite index such that $H^{\prime}$ is type-definable over some $e \in \operatorname{acl}(\emptyset)$.

Proof. We can replace $A$ by the name of $H$. So without loss of generality, $A$ is *-algebraic. Choose $b \in G$ m-generic over $A$. Let $d$ be the name of Hb. Clearly, $\operatorname{Tr}(b / A d)=\operatorname{Tr}(b / A) \cap H b$. So $\operatorname{Tr}(b / A d)$ is open in $H b$. By m-normality, choose $c \in \operatorname{acl}(b) \cap \operatorname{acl}(A d)$ with $b \stackrel{\mathrm{m}}{\Perp} A d(c)$. We can assume that $\operatorname{Tr}(b / c) \subseteq \operatorname{Tr}(b / A d)$. So $\operatorname{Tr}(b / c)$ is open in $H b$. 
Let $q=\operatorname{tp}(b / c)$. By Theorem 4.3, possibly expanding $c$ a little, we can assume that $q(\mathfrak{C})$ is a right coset of $H_{c}=\operatorname{Stab}(q(\mathfrak{C}))$. Clearly, $H_{c}$ is typedefinable over $c$ and is open in $H$, hence it has finite index in $H$.

Say, $H_{c}=H \cap U$ for some clopen $U \subseteq G$. Let $a$ be the name of $U, a \in$ $\operatorname{acl}(\emptyset)$. Choose $b^{\prime} \equiv b(A a)$ with $b^{\prime}{ }^{\mathrm{m}} \downarrow b(A a)$. Hence $b^{\prime}{ }^{\mathrm{m}} \downarrow b$. Choose $c^{\prime}$ with $A a b c \equiv A a b^{\prime} c^{\prime}$ and let $H_{c^{\prime}}$ be the conjugate of $H_{c}$. Clearly, $H_{c}=H \cap U=H_{c^{\prime}}$,

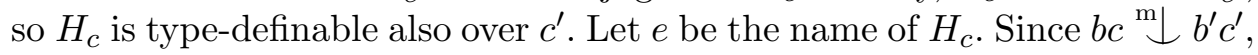
we see that $e \in \operatorname{acl}(\emptyset)$.

Corollary 4.5. Assume $T$ is small, m-normal and $G$ is a $*$-algebraic group. Then $G$ contains a clopen abelian subgroup of finite index.

Proof. For any $b \in G$ let $H_{b} \subseteq G \times G$ be the graph of the inner automorphism $x \mapsto b x b^{-1}$. Now let $b \in G$ be m-generic. Choose $H^{\prime}<H_{b}$ such that $H^{\prime}$ is open in $H$ and type-definable over an $e$ algebraic over $\emptyset$. Also, choose a clopen $U \subseteq G \times G$ with $H^{\prime}=H_{b} \cap U$. We can assume that $U$ is definable over $e$. Let $p=\operatorname{tp}(b / e)$. So $p(\mathfrak{C})$ is open in $G$. Let $V$ be the projection of $H^{\prime}$ to the first coordinate. So $V$ is an open subgroup of $G$. We shall prove that

$$
b_{1}^{-1} b_{2} \in C(V) \quad \text { for } b_{1}, b_{2} \in p(\mathfrak{C}) .
$$

Indeed, since $b_{1}, b_{2} \in p(\mathfrak{C})$, we have $H_{b_{1}} \cap U=H^{\prime}=H_{b_{2}} \cap U$, so for $x \in V$ we have $b_{1} x b_{1}^{-1}=b_{2} x b_{2}^{-1}$. Hence $b_{1}^{-1} b_{2} \in C(x)$.

By (a), $p(\mathfrak{C})^{-1} \cdot p(\mathfrak{C}) \subseteq C(V)$, hence $C(V)$ is a clopen subgroup of $G$. Clearly, $V \cap C(V)$ is an abelian open subgroup of finite index in $G$.

Corollary 4.6. If $T$ is superstable, with few countable models, and $G$ is a *-algebraic group, then $G$ is abelian-by-finite. In particular, if $H$ is a standard group type-definable in $T$, then the group $H / H^{0}$ is abelian-by-finite.

I do not know of any stable group $H$ for which the group $H / H^{0}$ is not abelian-by-finite. If such a group exists in a small stable theory $T$, then $T$ cannot be m-normal.

5. m-non-orthogonality. In this section we examine the notion of m-non-orthogonality of $*$-algebraic types. We show that it has properties similar to non-orthogonality in the theory of forking independence. Also, we analyze m-non-orthogonality among locally modular $*$-algebraic types of $\mathcal{M}$-rank 1 , in an m-normal theory. The next remark will facilitate the proofs in this section.

Remark 5.1. Assume $A$ is finite and $p, q \in S(A)$ are $*$-algebraic. Then $p$ and $q$ are $m$-orthogonal iff for every $b *$-algebraic over $A$, all $m$-free extensions of $p$ and of $q$ over Ab are almost m-orthogonal.

Proof. This follows from Lemma 1.9. 
Lemma 5.2. Assume $T$ is small, $m$-stable and $p, q \in S(\emptyset)$ are $*$-algebraic. Then $p$ is $m$-orthogonal to $q$ iff for all finite m-independent $I \subseteq p(\mathfrak{C})$ and all finite m-independent $J \subseteq q(\mathfrak{C})$, we have $I \stackrel{\mathrm{m} \downarrow}{\downarrow} J$.

Proof. $\Rightarrow$ is clear from definition.

$\Leftarrow$ Suppose $a \in p(\mathfrak{C}), b \in q(\mathfrak{C}), C$ is finite, $a^{\mathrm{m} \downarrow} C, b^{\mathrm{m}} \mathfrak{L} C$ and $a^{\mathrm{m} \mathscr{L}}$ $b(C)$. By Remark 5.1 we can assume that $C$ is $*$-algebraic. Suppose $a \stackrel{\mathrm{m}}{\perp} b$. Then $a b \stackrel{\mathrm{m} \chi}{ } C$. Choose an infinite sequence $\left\{a_{n} b_{n}\right\}_{n<\omega}$ of realizations of $\operatorname{tp}(a b / C), \mathrm{m}$-independent over $C$. Since $T$ is m-stable, the set $\left\{a_{n} b_{n}: n<\omega\right\}$ cannot be m-independent. On the other hand, each of $\left\{a_{n}\right\}_{n<\omega},\left\{b_{n}\right\}_{n<\omega}$ is m-independent over $C$. Since $a{ }^{\mathrm{m}} \downarrow C$ and $b^{\mathrm{m}} \mathfrak{L} C$, we conclude that each of $\left\{a_{n}\right\}_{n<\omega},\left\{b_{n}\right\}_{n<\omega}$ is m-independent. Hence for some $n, a_{<n}{ }_{\mathrm{m}} \mathcal{L} b_{<n}$.

LEMma 5.3. Assume $T$ is small, $m$-stable and $a$ and $p_{a} \in S(a)$ are *-

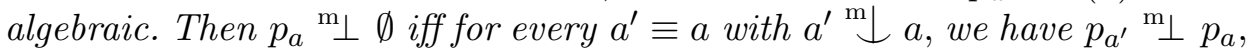
where $p_{a^{\prime}}$ is the conjugate of $p_{a}$.

Proof. $\Rightarrow$ Let $q_{a}, q_{a^{\prime}} \in S\left(a a^{\prime}\right)$ be m-free extensions of $p_{a}, p_{a^{\prime}}$ respectively. It is enough to show that $q_{a}{ }^{\mathrm{m}} \perp q_{a^{\prime}}$. Let $I \subseteq q_{a}(\mathfrak{C}), J \subseteq q_{a^{\prime}}(\mathfrak{C})$ be finite m-independent sequences. By Lemma 5.2 it is enough to show that $I \stackrel{\mathrm{m}}{\downarrow}$ $J\left(a a^{\prime}\right)$.

Now $J^{\mathrm{m}} \downarrow$ are done.

$\Leftarrow$ Suppose $p_{a}$ is m-non-orthogonal to some $q \in S(A)$ which is $\mathrm{m}$-free over $\emptyset$. By Lemma 5.2 choose finite m-independent sequences $I \subseteq p_{a}(\mathfrak{C})$ and $J \subseteq q(\mathfrak{C})$ with $I{ }^{\mathrm{m}} \mathcal{L} J(a)$. Hence $a I^{\mathrm{m}} \downarrow J$. Take an m-independent sequence $\left\{a_{n} I_{n}\right\}_{n<\omega}$ of realizations of $\operatorname{tp}(a I / J)$. Then $\left\{a_{n} I_{n}\right\}_{n<\omega}$ is m-independent over $\emptyset$ (by m-orthogonality of $p_{a}$ and $p_{a^{\prime}}$ ) and $a_{n} I_{n}{ }^{\mathrm{m}} \mathcal{L} J$, contradicting m-stability.

Now we turn to m-normal theories and $*$-algebraic types of $\mathcal{M}$-rank 1 . For such types, Lemmas 5.2 and 5.3 remain true without the m-stability assumption.

Lemma 5.4. Assume $T$ is small and m-normal.

(1) If $p, q \in S(\emptyset)$ are $*$-algebraic of $\mathcal{M}$-rank 1 , then $p{ }^{\mathrm{m}} \perp q \Leftrightarrow$ for all finite $m$-independent $I \subset p(\mathfrak{C})$ and all finite m-independent $J \subseteq q(\mathfrak{C})$, we have $I^{\mathrm{m}} \downarrow$ J.

(2) If $a$ and $p_{a} \in S(a)$ are $*$-algebraic and $\mathcal{M}\left(p_{a}\right)=1$, then $p_{a}{ }^{\mathrm{m}} \perp \emptyset$ iff $p_{a}{ }^{\mathrm{m}} \perp p_{a^{\prime}}$ for every $a^{\prime} \equiv$ a with $a \stackrel{\mathrm{m}}{\perp} a^{\prime}$, where $p_{a^{\prime}}$ is the conjugate of $p_{a}$.

Proof. $(1) \Rightarrow$ is obvious. For $\Leftarrow$ we repeat the proof of Lemma 5.2. Keeping the notation from that proof, notice that it is enough to show that if

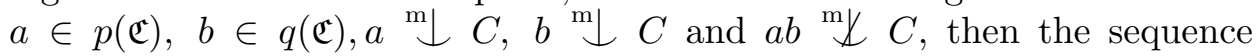
$\left\{a_{n} b_{n}\right\}_{n<\omega}$ cannot be m-independent. 
Suppose that it is. By m-normality, for each $n$ choose a $c_{n} \in \operatorname{acl}\left(a_{n} b_{n}\right) \cap$ $\operatorname{acl}(C)$ with $a_{n} b_{n}{ }^{\mathrm{m}} \mathfrak{L} C\left(c_{n}\right)$, so that all the $c_{n}$ 's realize the same type $r \in S(\emptyset)$. By the Lascar inequalities, $\mathcal{M}(r)=1$. By m-independence of $\left\{a_{n} b_{n}\right\}$, we conclude that $c_{n}, n<\omega$, are m-independent, and $c_{n} \in \operatorname{acl}(C)$. This contradicts Remark 2.10.

(2) The proof of $\Rightarrow$ is the same as in Lemma 5.3. For $\Leftarrow$, suppose $p_{a}$ is m-non-orthogonal to some $q \in S(\emptyset)$. This means that for some finite $B$ containing $a$, some m-free extensions $p^{\prime}$ and $q^{\prime}$ over $B$ of $p_{a}$ and $q$ respectively are not almost m-orthogonal. Without loss of generality, $B$ is $*$-algebraic, so we may assume $B=a$. The rest of the proof is as before, with modifications similar to those in (1), only now the sets $I, J$ have size 1.

In the theory of forking, if $p, q \in S(\emptyset)$ are stationary regular locally modular types and $p \not \subset q$, then $p^{2} a_{\not} q^{2}$. Also, for any $a \models p$, the type $p \mid a$ is modular. I was unable to bound the size of the sets $I, J$ appearing in Lemma 5.4(1). Also, it is not clear which object should play the role of a modular type in the context of m-independence and *-algebraic types. It seems that $*$-algebraic groups of $\mathcal{M}$-rank 1 behave like modular types.

TheOREm 5.5. Assume $T$ is small, m-normal and $G, H$ are $m$-nonorthogonal *-algebraic groups of $\mathcal{M}$-rank 1 , type-definable over $\emptyset$. Then locally $G$ and $H$ are definably isomorphic (over some parameter from acl $(\emptyset)$ ).

Proof. Choose a finite set $B$ and elements $a \in G$ and $b \in H$, m-generic over $B$, such that $a{ }^{\mathrm{m}} \not{L} b(B)$. We can assume that $a$ and $b$ are interdefinable over $B$ and the set of realizations of $\operatorname{tp}(a b / B)$ in $G \times H$ is a coset of the subgroup $S=\operatorname{Stab}(\operatorname{tp}(a b / B)) \subseteq G \times H$. Choose $S^{\prime}<S$ of finite index in $S$ and type-definable over some $e \in \operatorname{acl}(\emptyset)$, provided by Theorem 4.4. Clearly $S^{\prime}$ is the graph of an $e$-definable isomorphism between some clopen subgroups of $G$ and $H$.

Theorem 5.5 improves [Ne5, Theorem 4.10], where we obtained only a local isomorphism between $G$ and $H$ definable over some parameters not necessarily in $\operatorname{acl}(\emptyset)$.

We end this paper stating some open problems. Fortunately, in small superstable theories with few countable models every *-algebraic type of $\mathcal{M}$-rank 1 is m-non-orthogonal to a $*$-algebraic group of $\mathcal{M}$-rank 1 (which, in virtue of Theorem 5.5, may play the role of a "modular type"). It is not clear if this is true in any small m-normal theory $\left({ }^{1}\right)$. Hrushovski [Hr] used germs of definable functions to construct, starting from a locally modular non-trivial regular type $p$, a definable regular group non-orthogonal to $p$. In an m-normal theory any *-algebraic non-trivial $\mathcal{M}$-rank 1 type $q$ is lo-

$\left({ }^{1}\right)$ In the meantime I proved this: see my paper Small profinite structures, to appear in Trans. Amer. Math. Soc. 
cally modular, so it would be natural to expect that some modification of Hrushovski's argument would work here, yielding a $*$-algebraic $\mathcal{M}$-rank 1 group m-non-orthogonal to $q$. However this approach does not work, because in m-independence we lack the notion of a stationary type. Still, the above idea of Hrushovski influenced me in the proof of Theorem 4.3.

Hrushovski used an analogue of Theorem 4.3 to describe forking on $G^{0}$ in terms of pseudo-endomorphisms, for a regular locally modular stable group $G$. Here we cannot expect a similar result, since *-algebraic groups have trivial connected components. In model theory there is a long list of results showing that a group minimal in some respect is abelian, or abelianby-finite. Here we proved that in a small m-normal theory any *-algebraic group $G$ is abelian-by-finite. Still, if we restrict just to $G$ of $\mathcal{M}$-rank 1 (which is a kind of minimality assumption), can we omit the m-normality assumption in the proof that $G$ is abelian-by-finite? I am pessimistic about that, despite the fact that by the results of Baudisch [Ba], for any standard group $G$ interpretable in a superstable theory, the group $G / G^{0}$ is solvable-by-finite, and if moreover $\mathcal{M}\left(G / G^{0}\right)=1$, then $G / G^{0}$ is abelian-by-finite. But, again, I know of no small theory which is not m-normal.

\section{References}

[Ba] A. Baudisch, On superstable groups, J. London Math. Soc. (2) 42 (1990), 452-464.

[Bu1] S. Buechler, Locally modular theories of finite rank, Ann. Pure Appl. Logic 30 (1986), 83-95.

[Bu2] -, On non-trivial types of U-rank 1, J. Symbolic Logic 52 (1987), 548-551.

[Hr] E. Hrushovski, Locally modular regular types, in: Classification Theory (Chicago, 1985), J. T. Baldwin (ed.), Springer, 1987, 132-164.

[HP] E. Hrushovski and A. Pillay, Weakly normal groups, in: Logic Colloquium 1985, North-Holland, Amsterdam, 1987, 233-244.

[Ne1] L. Newelski, A proof of Saffe's conjecture, Fund. Math. 134 (1990), 143-155.

[Ne2] -, Meager forking, Ann. Pure Appl. Logic 70 (1994), 141-175.

[Ne3] -, M-rank and meager groups, Fund. Math. 150 (1996), 149-171.

$[\mathrm{Ne} 4]-, \mathcal{M}$-gap conjecture and m-normal theories, Israel J. Math. 106 (1998), 285-311.

[Ne5] -, Geometry of *-finite types, J. Symbolic Logic 64 (1999), 1375-1395.

[Ne6] - Flat Morley sequences, ibid., 1261-1279.

[Ox] J. C. Oxtoby, Measure and Category, Springer, Berlin, 1980.

[Pi] A. Pillay, Geometric Stability Theory, Oxford Univ. Press, 1996.

Mathematical Institute

Wrocław University

50-384 Wrocław, Poland

E-mail: newelski@math.uni.wroc.pl
Mathematical Institute Polish Academy of Sciences 51-617 Wrocław, Poland

Received 12 June 1998;

in revised form 29 December 2000 